\title{
Changements hydrographiques et volcanisme plio-quaternaire dans les bassins de la Loire et de l'Allier (Massif central, France)
}

\section{Hydrographic Changes and Plio-Quaternary Volcanism in the Drainage Basins of the Loire and Allier Rivers (Massif Central, France)}

\section{Hydrographische Veränderungen und Plio-Quaternär-Vulkanismus in den Becken von Loire und Allier (Massif Central, Frankreich)}

\author{
Jean-François Pastre, Emmanuelle Defive, Frédérique Gablier et Yannick \\ Lageat
}

Volume 51, numéro 3, 1997

URI : https://id.erudit.org/iderudit/033129ar

DOI : https://doi.org/10.7202/033129ar

Aller au sommaire du numéro

Éditeur(s)

Les Presses de l'Université de Montréal

ISSN

0705-7199 (imprimé)

1492-143X (numérique)

Découvrir la revue

Citer cet article

Pastre, J.-F., Defive, E., Gablier, F. \& Lageat, Y. (1997). Changements hydrographiques et volcanisme plio-quaternaire dans les bassins de la Loire et de l'Allier (Massif central, France). Géographie physique et Quaternaire, 51(3), 295-314. https://doi.org/10.7202/033129ar

\section{Résumé de l'article}

L'analyse géométrique des paléotracés des bassins supérieurs de la Loire et de l'Allier précise leur paléogéographie et définit leurs relations avec le volcanisme et les phénomènes sédimentaires associés. Les données radiométriques récentes et les marqueurs téphrochronologiques fournissent un cadre chronologique à ce schéma. Ces données rendent peu vraisemblable l'hypothèse d'un cours plio-pléistocène de la Loire vers l'Allier. Elles démontrent en contrepartie l'existence probable d'un ancien tracé méridien de la rivière fossilisé par les basaltes du Devès à l'amont du bassin du Puy. Dans le haut bassin de l'Allier, les preuves d'un passage de l'Allier par le bassin de Paulhaguet au Pliocène demeurent contestables. L'existence d'un ancien tracé N-S de la Sénouire, barré par l'appareil volcanique du Pié-du-Roi. est par contre confirmée. Elle peut être considérée comme à l'origine de la sédimentation dans le bassin et du déversement de la rivière vers le nord. Plus au nord, la paléogéographie de la vallée pliocène de l'Allagnon, fossilisée par les basanites du Cézallier, est précisée. À la bordure orientale du Mont-Dore, des modifications de tracés importantes sont mises en évidence dans les drainages quaternaires des vallées affluentes de l'Allier (Couzes). Enfin, à l'est de ce secteur, l'évolution complexe du tracé de l'Allier est retracée avec précision sur plus de $4 \mathrm{Ma}$, grâce aux jalons fournis par le volcanisme. Ces résultats montrent le rôle direct du volcanisme dans la modification de la majorité des tracés, mais soulignent aussi son rôle inducteur important dans les sédimentations fluvio-lacustres qui leur sont liées. Ils conduisent ainsi à réduire le rôle direct de la tectonique, au profit de celui des dynamiques climatiques exacerbées par le volcanisme. 


\title{
CHANGEMENTS HYDROGRAPHIQUES ET VOLCANISME PLIO-QUATERNAIRE DANS LES BASSINS DE LA LOIRE ET DE L'ALLIER (MASSIF CENTRAL, FRANCE)
}

\begin{abstract}
Jean-François PASTRE*, Emmanuelle DEFIVE, Frédérique GABLIER et Yannick LAGEAT; $1^{\text {er }}$ auteur : Laboratoire de Géographie physique, URA 141 CNRS et Université de Paris I, 1, place Aristide Briand, 92195 Meudon Cedex, France, et membre associé à l'UPRES-A 1562, Clermont-Ferrand ; $2^{\circ}$ et $4^{\circ}$ auteurs : Géodynamique des milieux naturels et anthropisés, UPRES-A 1562, CNRS et Université Blaise Pascal, 29 boulevard Gergovia, F63037 Clermont-Ferrand Cedex 1, France ; $3^{\mathrm{e}}$ auteure : Cour Malo, 2, rue Bernard Duplessis, 77520 Donnemarie-Dontilly, France.
\end{abstract}

RÉSUMÉ L'analyse géométrique des paléotracés des bassins supérieurs de la Loire et de l'Allier précise leur paléogéographie et définit leurs relations avec le volcanisme et les phénomènes sédimentaires associés. Les données radiométriques récentes et les marqueurs téphrochronologiques fournissent un cadre chronologique à ce schéma. Ces données rendent peu vraisemblable l'hypothèse d'un cours plio-pléistocène de la Loire vers l'Allier. Elles démontrent en contrepartie l'existence probable d'un ancien tracé méridien de la rivière fossilisé par les basaltes du Devès à l'amont du bassin du Puy. Dans le haut bassin de l'Allier, les preuves d'un passage de l'Allier par le bassin de Paulhaguet au Pliocène demeurent contestables. L'existence d'un ancien tracé N-S de la Sénouire, barré par l'appareil volcanique du Pié-du-Roi, est par contre confirmée. Elle peut être considérée comme à l'origine de la sédimentation dans le bassin et du déversement de la rivière vers le nord. Plus au nord, la paléogéographie de la vallée pliocène de l'Allagnon, fossilisée par les basanites du Cézallier, est précisée. À la bordure orientale du Mont-Dore, des modifications de tracés importantes sont mises en évidence dans les drainages quaternaires des vallées affluentes de l'Allier (Couzes). Enfin, à l'est de ce secteur, l'évolution complexe du tracé de l'Allier est retracée avec précision sur plus de 4 $\mathrm{Ma}$, grâce aux jalons fournis par le volcanisme. Ces résultats montrent le rôle direct du volcanisme dans la modification de la majorité des tracès, mais soulignent aussi son rôle inducteur important dans les sédimentations fluvio-lacustres qui leur sont liées. Ils conduisent ainsi à réduire le rôle direct de la tectonique, au profit de celui des dynamiques climatiques exacerbées par le volcanisme.

Manuscrit reçu le 8 octobre 1996 ; manuscrit révisé accepté le 14 avril 1997
ABSTRACT Hydrographic changes and Plio-Quaternary volcanism in the drainage basins of the Loire and Allier rivers (Massif Central, France). Geometric analysis was used to determine the paleogeography of the valley courses in the upper drainage basins of the Loire and Allier rivers, and to define their relations to regional volcanism and associated sedimentation dynamics. The chronology of this scheme is based on the integration of new radiometric data and the study of tephrochronological indicators. According to these data, there is no evidence for a Plio-Pleistocene course of the Loire towards the Allier. On the contrary, a probable S-N water course of the river is fossilized by the Devès basalts upstream from the basin of Le Puy. In the upper Allier drainage basin there are insufficient arguments to prove that the Allier ever flowed through the Paulhaguet basin during Pliocene. However, our data confirm the existence of an old N-S course of the Sénouire, now dammed by the Pié-duRoi volcano. It explains the sedimentation in the basin and the change of direction of the course to the north. Further north, Pliocene paleogeography of the Allagnon, buried under the Cézallier basanites, is examined. On the eastern slopes of the Mont Dore, important changes in courses are evident within the Quaternary network of the tributaries of the Allier (Couzes). Finally, further east, the $4 \mathrm{Ma}$ complex evolution of the Allier course can be described precisely, using volcanic chronological indicators. Our results illustrate the direct role played by volcanism in the changes of the river courses and its important role in the fluvio-lacustrine sedimentation. The part played by tectonics seems to be less important than that of the climatic dynamic.
ZUSAMMENFASSUNG Hydrographische Veränderungen und Plio-QuaternärVulkanismus in den Becken von Loire und Allier (Massif Central, Frankreich). Die geometrische Analyse des Paläoverlaufs der oberen Becken der Loire und des Allier verdeutlicht ihre Paläogeographie und bestimmt ihre Beziehungen zum Vulkanismus und damit verbundenen Sedimentationssphänomenen. Die neuen radiometrischen Daten und die tephrochronologischen Indikatoren geben diesem Schema einen chronologischen Rahmen. Die Hypothese eines Verlaufs der Loire zum Allier hin im PlioPleistozän erscheint durch diese Daten unwahrscheinlich. Dagegen belegen sie die Wahrscheinlichkeit eines alten südlichen Verlaufs des durch die Devès-Basalte versteinerten Flusses oberhalb des Beckens von Puy. Im oberen Allier-Becken bleiben die Beweise dafür, dass der Allier im Pliozän das Becken von Paulhaguet durchfloss, anfechtbar. Jedoch wird ein alter N-S-Verlauf der Sénouire bestätigt, der jetzt durch den Pié-du-Roi-Vulkan abgeschnitten wird. Das führte zur Sedimentation im Becken und der Veränderung des Flusslaufs nach Norden. Weiter nördlich wird die Paleogeographie des Allagnon-Pliozän-Tals, das durch die Cézallier-Basanite versteinerte, präzisiert. Am östlichen Rand des Mont-Dore werden wichtige Veränderungen der Läufe in den Quartär-Einzugsgebieten der Zuflüsse des Allier (Couzes) dargelegt. Schliesslich wird im Osten dieses Gebiets mit Hilfe der durch den Vulkanismus gelieferten Anhaltspunkte die komplexe Entwicklung des Laufs des Allier über mehr als $4 \mathrm{Ma}$ präzise nachvollzogen. Diese Ergebnisse zeigen die direkte Einwirkung des Vulkanismus auf die Veränderung der Mehrzahl der Läufe ; sie heben aber auch seine wichtige auslösende Wirkung in den mit ihnen verbundenen FlussSee-Sedimentationen hervor. Die Ergebnisse führen zu einer Minderung der direkten Rolle der Tektonik zugunsten der durch den Vulkanismus verschärften Klima-Dynamik. 


\section{INTRODUCTION}

Le volcanisme plio-quaternaire du Massif central a entraîné des perturbations importantes du réseau hydrographique tributaire des bassins supérieurs de l'Allier et de la Loire (fig. 1). Les épanchements basaltiques du Cézallier et du Devès et les émissions pyroclastiques du Mont-Dore ont modifié les rythmes de creusement des vallées, tout en entraînant des modifications plus ou moins importantes de leurs tracés. Malgré les progrès réalisés dans l'analyse de ces modifications, de nombreuses imprécisions subsistent. Elles concernent aussi bien leur paléogéographie que leur dynamique ou

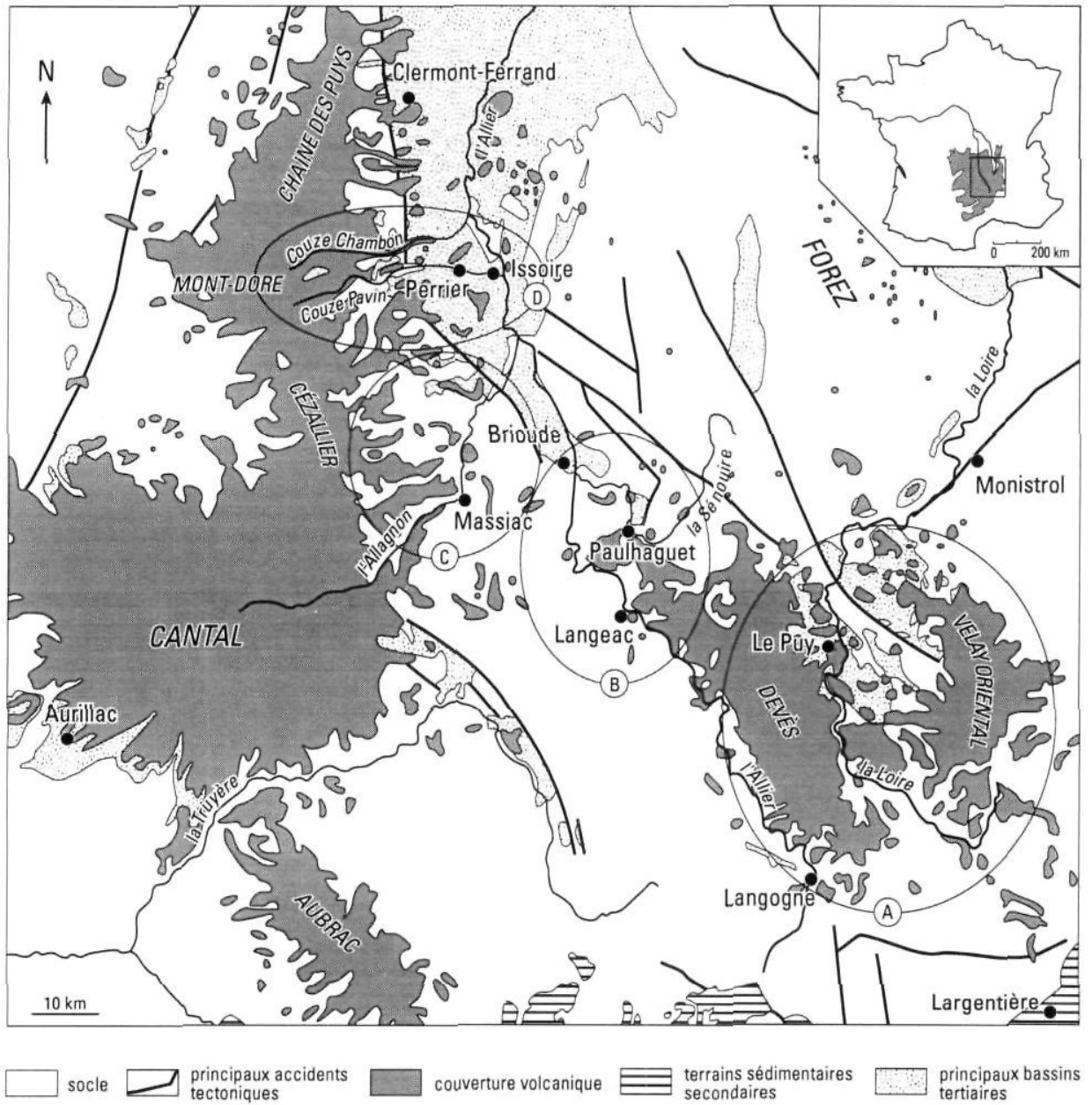

A. bassin supérieur de la Loire ; B. Allier, Sénouire et bassin de Paulhaguet; C. vallée de l'Allagnon et Cézallier ; D. régions des Couzes et de Perrier.

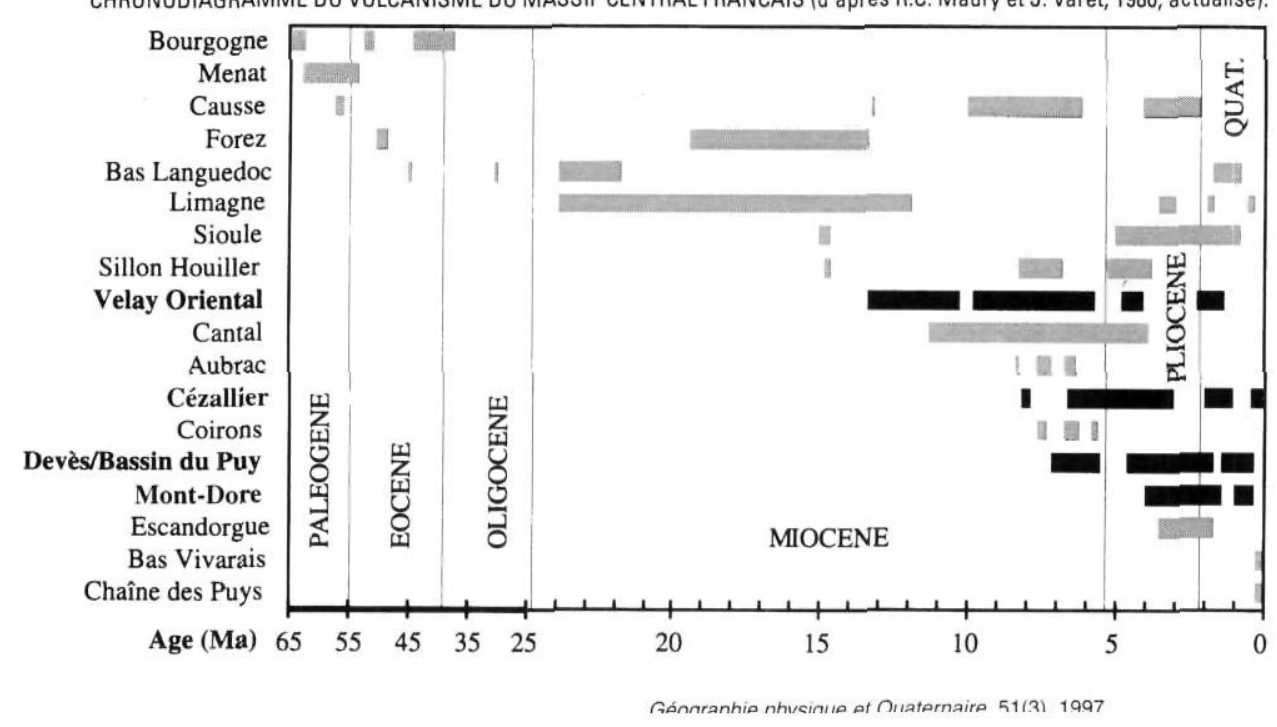

FIGURE 1. Carte de localisation des secteurs étudiés et position chronologique au sein du volcanisme du Massif central.

Location map of the studied areas and position in the chronology of the Massif Central. 
leur chronologie. Si une majorité d'exemples bénéficient de preuves solides, d'autres, et non des moindres, comme la translation Loire-Allier ou le passage de l'Allier par le bassin de Paulhaguet, demeurent des sujets de controverse. Quant à l'interprétation dynamique des faits, elle s'appuie souvent insuffisamment sur l'analyse géométrique des formes et des formations. Différents stades d'évolution sont parfois confondus pour servir une même hypothèse, alors que les données de terrain les distinguent. Ce travail présente une nouvelle analyse paléogéographique des changements concernés. II en propose une chronologie rénovée, établie à partir des datations radiométriques et des données téphrochronologiques. Enfin, il analyse leurs liaisons avec le volcanisme.

\section{MÉTHODOLOGIE}

Ce travail est fondé sur une analyse de terrain détaillée, comportant une circonscription précise des paléoformes et des formations associées (alluvions, pyroclastites, coulées basaltiques). Cette analyse est complétée par une interprétation des données de sondages réalisés dans le cadre de projets d'aménagement. Les corrélations sont assurées par une étude pétrographique et minéralogique systématique des formations (Pastre, 1987 ; Defive, 1996 ; Pastre, inédit), reposant en particulier sur une analyse des cortèges de minéraux lourds et la recherche de marqueurs symptomatiques de l'activité volcanique. La chronologie est définie en fonction de la téphrochronologie établie pour le strato-volcan du Mont-Dore ainsi que par différentes datations radiométriques (potassium-argon, argon-argon) réalisées sur les coulées basaltiques dans le cadre de travaux antérieurs ou liés à ce travail (Cantagrel in Defive, 1996).

\section{LES MODIFICATIONS DE TRACÉ DU RÉSEAU HYDROGRAPHIQUE ET LE VOLCANISME DU DEVÈS DANS LE HAUT BASSIN DE LA LOIRE}

Dans le haut bassin de la Loire, les plateaux du Velay oriental recèlent la trace du passage d'un écoulement SENO qui pourrait représenter l'ancêtre miocène de la Loire. La fixation définitive des grandes lignes du réseau hydrographique actuel remonte cependant au Pliocène inférieur, voire au Miocène supérieur ( 7 à $8 \mathrm{Ma}$ ). Si l'hypothèse d'un passage de la Loire vers l'Allier à la charnière plio-pléistocène apparaît peu vraisemblable, cette période n'en représente pas moins le début d'une évolution fortement perturbée par le volcanisme, tant en ce qui concerne les tracés qu'en ce qui à trait à l'encaissement.

\section{1) UN TRACÉ MIOCĖNE DE LA LOIRE FOSSILISÉ SOUS LES LAVES DES HAUTS PLATEAUX DU VELAY ORIENTAL}

Les laves du Velay oriental ont fossilisé sur les hauts plateaux, contournés aujourd'hui par la Loire, les matériaux résiduels d'épandages fluviatiles grossiers. D'après la répartition des points d'affleurement, ceux-ci pourraient jalonner le passage d'un écoulement ancien assimilable à un ancêtre finimiocène de la Loire actuelle. La nature du matériel, essentiellement constitué de galets de quartz et de chailles, implique un sens d'écoulement du SE vers le NO (fig. 2). Les conséquences de ces observations sont importantes. Les matériaux en question indiquent qu'un système de pentes régional orienté vers le NO existait déjà à cette époque, avant les principales dislocations de la fin du Miocène et alors que le socle du Massif central n'était pas encore déconnecté vers l'est de sa bordure sédimentaire secondaire.

\section{2) LA FIXATION DÉFINITIVE DU RÉSEAU HYDROGRAPHIQUE ET L'ÉVOLUTION PLIOCĖNE}

Ce n'est cependant qu'au terme de la construction du massif volcanique du Velay oriental et alors qu'un nouvel agencement régional des pentes était né des dislocations de la fin du Miocène, que le réseau hydrographique, jusque là très perturbé, a pu se réorganiser. Jalonnées par les dernières coulées du Velay oriental, les premières marques de cette renaissance, vallée ample aux versants largement évasés, pourraient remonter à la charnière mio-pliocène ou au Miocène supérieur, et en tout cas de façon certaine au Pliocène inférieur. De cette époque, située aux environs de 7 à $8 \mathrm{Ma}$, date donc la fixation du réseau hydrographique actuel. Les rares coulées situées sur le tracé de la Loire (Camp d'Antonne : 6,8 0 0,2 Ma, Cantagrel in Defive, 1996 ; coulées épanchées en rive gauche sur les hauts versants qui dominent le site de Lafarre : 2,77 $\pm 0,07 \mathrm{Ma}$, ibid.) constituent des jalons majeurs dans l'histoire du creusement. La position de la coulée du Camp d'Antonne, perchée à $250 \mathrm{~m}$ au-dessus du talweg actuel à hauteur d'Arlempdes, permet ainsi de reconstituer la trace d'un premier profil en long de la rivière (fig. 3). Celle-ci empruntait une large vallée, déjà légèrement inscrite dans le horst de Chaspinhac au même emplacement que les gorges actuelles (fig. 2).

Jusqu'aux alentours de 2 à $3 \mathrm{Ma}$, l'évolution pliocène voit s'encaisser sur place les cours d'eau précédemment fixés. Tandis que la morphologie de la vallée de la Loire évoluait vers des berceaux de plus en plus profonds, aux versants plus pentus, l'encaissement, d'une valeur maximale de $300 \mathrm{~m}$ (1250 m à $950 \mathrm{~m}$ ), s'est effectué au rythme d'un exhaussement saccadé et en fonction d'un compartimentage serré des blocs cristallins juxtaposés.

\section{3) LES CHANGEMENTS À LA CHARNIÈRE PLIO-PLÉISTOCÈNE}

Au calme de l'évolution pliocène succède, à la charnière plio-pléistocène, une histoire mouvementée. La dégradation bioclimatique prétiglienne, mise en évidence grâce à la séquence lacustre de Ceyssac (Ablin, 1985, 1991) pourrait être à l'origine du changement qui s'opère dans la morphologie de la vallée. Le profil passe alors du berceau à la gorge. Une coulée épanchée dans la vallée à Issarlès $(1,96 \pm 0,09 \mathrm{Ma}$, Cantagrel in Defive, 1996) a ainsi fossilisé la trace de ce passage, vraisemblablement progressif dans les formes, mais rapide dans le temps (fig. 3 ).

\section{a) Les changements de détail du tracé de la Loire en amont d'Arlempdes}

L'étude de la section amont de la vallée jusqu'à Arlempdes conduit à distinguer dans le creusement de la gorge plio- 


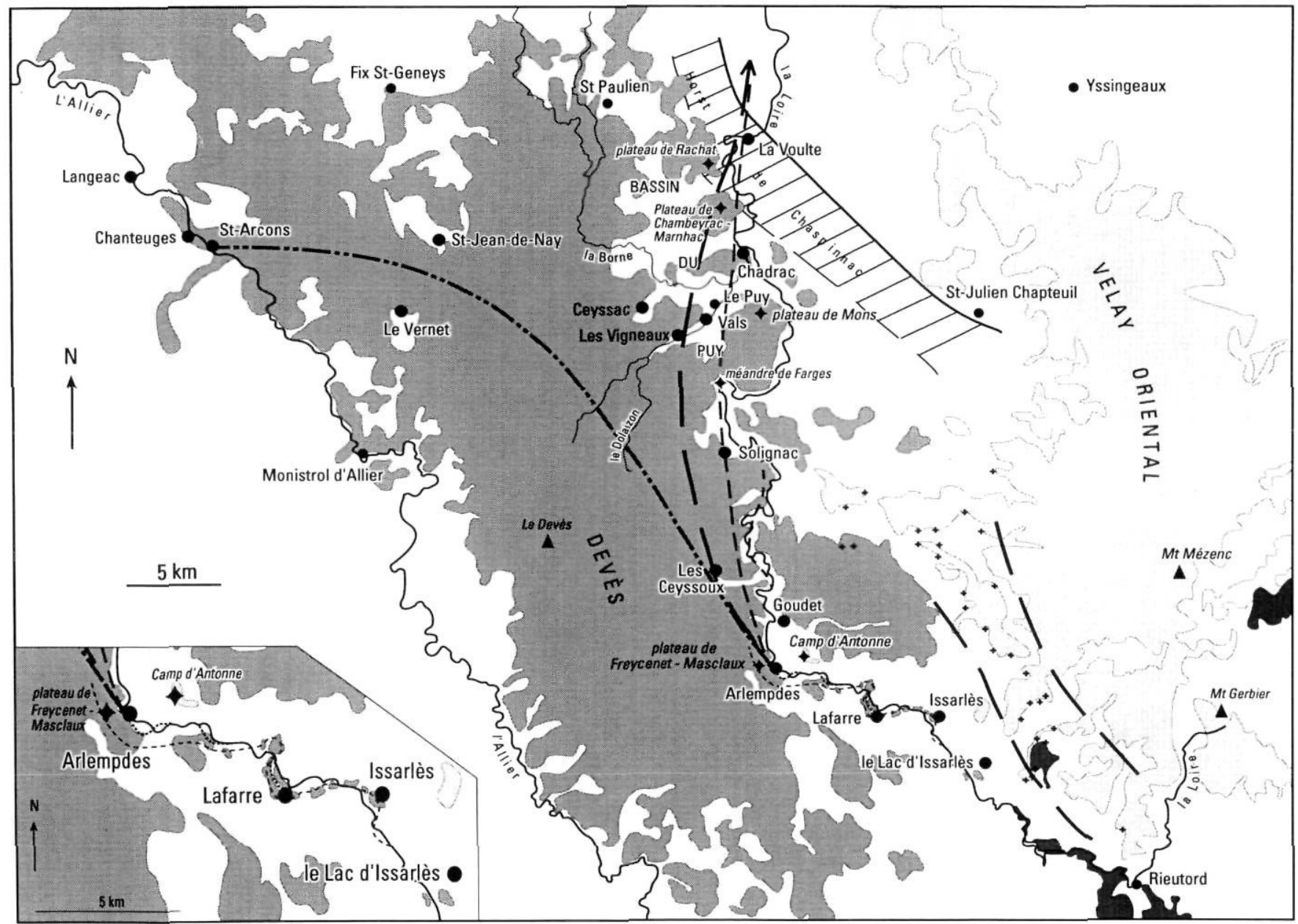

\section{Géologie}

substratum cristallin et paléogène volcanisme ancien du Velay oriental volcanisme du Devès

volcanisme récent du haut-Vivarais

\section{Hydrographie}

$\checkmark$ réseau hydrographique actuel

_ points d'affleurement

+++ d epandages fluviatiles jalonnant un ancêtre de la Loire

tracé de la Loire anté-

villafranchienne d'après $J$. Crosnier-Leconte niveau d'écoulement jalonné par la coulée d'Issarlès niveau d'écoulement jalonné par les coulées de Lafarre

\section{paléochenal plio-pléistocène} de la Loire vers $2 \mathrm{Ma}$

paléochenal plio-pléistocène de la Loire entre 2 et $1 \mathrm{Ma}$ niveau d'écoulement contemporain du creusement de la gorge actuelle $(<1 \mathrm{Ma}$

Divers

- localités et sites évoqués dans le texte

- autres localités

\ principaux sommets

FIGURE 2. Les changements hydrographiques dans le haut bassin de la Loire au Pliocène et au Pléistocène.

Changes in the rivers courses in the upper basin of the Loire during the Pliocene and the Pleistocene. 
pléistocène trois étapes. D'après les datations effectuées à Lafarre (Cantagrel, 1984, in Fouris, 1989), celles-ci se situent toutes aux environs de $2 \mathrm{Ma}$. Dans ce secteur, le creusement a été interrompu à trois reprises par l'épanchement de coulées dites de génération "Issarlès ", "Lafarre " et "Arlempdes " (fig. 3). À chaque fois le cours d'eau a dû réentailler des épaisseurs importantes de 50 à $150 \mathrm{~m}$ de basalte, avant de poursuivre son encaissement. Des changements de tracé s'en sont suivis. Ils ont cependant toujours été faibles, compte tenu de la contrainte latérale imposée par la présence des hauts versants cristallins. Au cours de cette évolution, l'encaissement a progressé de $150 \mathrm{~m}$ par rapport au niveau du dernier berceau pliocène $(950 \mathrm{~m})$. Là encore, c'est l'exhaussement de tout le domaine amont qui a motivé le creusement au-delà d'un axe de fracture que les derniers rejeux vers 2 Ma permettent de localiser sous les laves du plateau de Freycenet Masclaux, entre Arlempdes et Goudet (fig. 2).

La gorge fossilisée sous les coulées de ce plateau correspond au plus bas niveau atteint par le talweg pliopléistocène de la Loire dans la section amont. Son creusement a été interrompu par les épanchements massifs qui marquent vers $2 \mathrm{Ma}$ le premier épisode majeur de la construction du plateau du Devès. À partir de ce moment, les gorges creusées dans la section amont n'ont plus évolué et ont même été remplies par un remblaiement fluvio-lacustre mis en place en arrière du barrage volcanique d'Arlempdes. Leur exhumation au cours du dernier million d'années a coïncidé avec le creusement de la gorge épigénique actuelle en aval d'Arlempdes.

b) Discussion sur l'hypothèse d'une translation Loire - Allier

La gorge plio-pléistocène de la Loire fossilisée sous le plateau de Freycenet - Masclaux a été mise en évidence dans les années 1940-1950, lors de la campagne de sondages effectuée en vue de l'implantation d'un barrage E.D.F. aux environs d'Arlempdes. Reprenant une discussion déjà ancienne (Robert, 1836 ; Baulig, 1928), Crosnier-Leconte $(1948,1956)$ a cru trouver dans ces résultats les arguments en faveur d'un passage de la Loire vers l'Allier. Cette hypothèse a été successivement réexaminée par Bout (1980), Larue (1979, 1982) et Étienne (1982).

Pour Crosnier-Leconte «l'ancienne Loire [...] passait à l'ouest des Ceyssoux, traversait probablement le plateau actuel du Velay entre Saint-Jean-de-Nay et Le Vernet [et aboutissait] dans la vallée actuelle de l'Allier vers Chanteuges Langeac " (Crosnier-Leconte, 1956). L'argumentation proposée par cet auteur peut être discutée. Comparer sur la base de la morphologie des secteurs aussi éloignés que ceux d'Arlempdes et Saint-Jean-de-Nay - Le Vernet - Saint-Arcons paraît d'autant plus risqué qu'il existe des dislocations contemporaines de l'édification du plateau du Devès et que le tracé potentiel est largement masqué par les basaltes. Parmi les autres arguments proposés, l'infléchissement “ inexpliqué " des coulées basaltiques dans le secteur de SaintArcons, ne tient pas compte du rôle joué par l'Allier et ses affluents, la Desges et surtout la Fioule ; l'élargissement " inexplicable " de l'Allier dans le même secteur, revient à omettre à nouveau le rôle de ces deux rivières ainsi que celui du bassin de Langeac immédiatement à l'aval. Enfin, comme le remarquent Larue (1982) et Pastre (1987), aucune corrélation satisfaisante ne peut être établie entre les nappes alluviales de ces deux domaines. Les alluvions de la Loire présentent des spectres de minéraux lourds à grenats, alors que ceux de l'Allier et de ses affluents à l'amont de Langeac, sont fortement enrichis par des apports locaux en staurotide (Pastre, 1987). Les alluvions de l'Allier ne comportent pas de phonolites du Mézenc, mais celles-ci sont aussi très rares dans les alluvions plio-quaternaires de la Loire. Quant au stock basaltique, il est ubiquiste et ne peut être utilisé. Les seuls traceurs utilisables que seraient les zircons du socle du haut bassin de la Loire ne se retrouvent pas dans les alluvions du haut bassin de l'Allier.

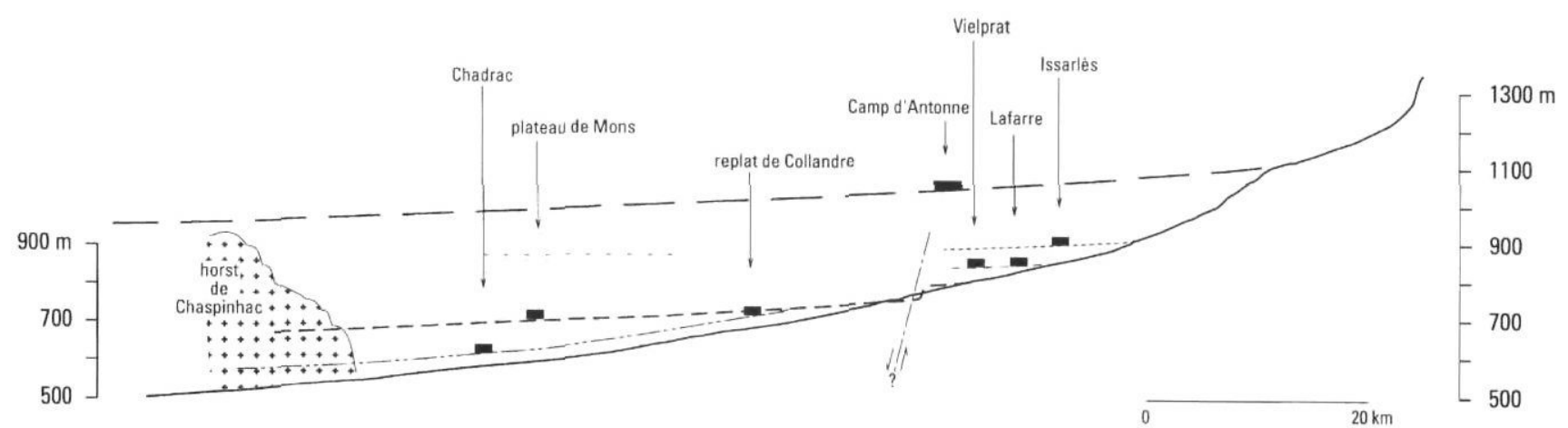

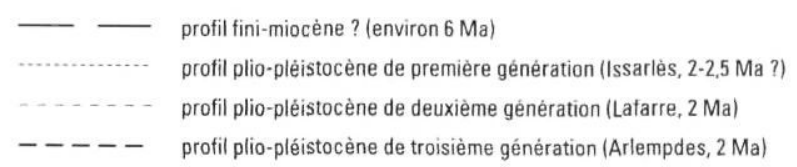

FIGURE 3. Évolution du profil en long de la Loire au PlioPléistocène.
Evolution of the longitudinal profile of the Loire during the PlioPleistocene. 
D'autres arguments vont également à l'encontre de l'hypothèse de Crosnier-Leconte : la composition des alluvions plio-quaternaires du bassin de la Borne témoigne selon Baulig (1928) d'un système de pentes déjà organisé à la charnière plio-pléistocène autour du bassin du Puy. Comme Boule (1893), cet auteur pense d'autre part que " la Loire pliocène s'échappait du bassin du Puy par le même chemin qu'aujourd'hui, c'est à dire par le défilé de Peyredeyre, entre Chadrac et Lavoûte-sur-Loire, lequel avait été creusé, dès cette époque, jusqu'à $80 \mathrm{~m}$ au-dessus de la rivière actuelle ". Ces hypothèses sont confirmées par les travaux en cours (Defive et Pastre).

Même en invoquant, comme le fait Crosnier-Leconte, un âge " anté-villafranchien » pour le paléochenal, l'hypothèse d'une liaison Loire - Allier ne tient pas si l'on admet que le horst de Chaspinhac porte déjà la trace du passage d'un niveau d'écoulement encore antérieur, établi à l'altitude de la coulée du Camp d'Antonne (fig. 3). L'âge des coulées de Lafarre indique par ailleurs que la gorge fossilisée à Arlempdes n'est pas antérieure à la charnière plio-pléistocène. Si Crosnier-Leconte n'a pu retrouver en direction du bassin du Puy la trace du paléochenal fossilisé sous le plateau de Freycenet - Masclaux, c'est qu'au-delà d'Arlempdes celui-ci est masqué en totalité sous les laves du Devès. Le coude opéré vers l'est par tous les affluents de rive gauche de la Loire entre Arlempdes et Solignac pourrait en marquer l'emplacement. La présence de dépôts phréatomagmatiques dans le ravin des Ceyssoux (fig. 2) pourrait le corroborer. Enfin, les alluvions grossières qui se retrouvent à la base et au sein des dépôts du remblaiement plio-pléistocène du bassin du Puy indiquent clairement l'existence d'un courant chargé et compétent venu du sud. L'hypothèse soutenue par CrosnierLeconte paraît ainsi très improbable. Celle d'un cours orienté vers le nord dès le Pliocène semble au contraire bénéficier d'un faisceau d'arguments favorables.

\section{c) Nature et portée de l'évolution paléogéographique du bassin du Puy}

Les observations effectuées dans le bassin du Puy conduisent à penser que l'altitude du talweg plio-pléistocène de la Loire y était proche de $700 \mathrm{~m}$. La charge grossière transportée par le cours d'eau se retrouve sous la forme d'une nappe à galets dont les points d'affleurement jalonnent le tracé de l'ancien chenal. À l'aval d'Arlempdes, celui-ci poursuivait son cours vers le nord à quelques kilomètres au plus à l'ouest de la gorge actuelle. La Loire arrivait sans doute dans le bassin du Puy par la dépression de Vals. L'épaisse nappe à galets (6 m), signalée par Boule (1893) aux Vigneaux, en serait la preuve. Elle passait ensuite probablement à l'emplacement du plateau de Mons où un banc à galets s'intercale entre les dépôts paléogènes et la coulée de base, et franchissait le horst à peu près par le même chemin qu'aujourd'hui (fig. 2).

Il est certain que la sédimentation plio-pléistocène du bassin du Puy, qui a abouti à la mise en place d'une série épaisse de 150 m au minimum, a pu être influencée par des affaissements tectoniques locaux, dont témoignent les pendages des dépôts dans certains secteurs (ex. Ceyssac). Cependant, la reconstitution des paléoprofils en long de la Loire entre le bassin du Puy, son amont et le horst de Chaspinhac prouve que les déformations tectoniques, d'une valeur maximale de 50 à $60 \mathrm{~m}$, ont de toute façon été insuffisantes pour permettre une telle accumulation. La présence de coulées épanchées à l'exutoire aval du bassin du Puy sur le horst de Chaspinhac dans l'axe supposé de la paléo-Loire (plateau de Rachat) et à son amont immédiat (plateau de Chambeyrac-Marhac), permet par ailleurs de se poser la question du rôle déterminant d'un barrage volcanique dont les étapes d'édification et de destruction peuvent être reconstituées en rapport avec l'évolution du creusement de la vallée de la Loire (Defive, 1996).

Après que la construction du plateau de Chambeyrac Marnhac ait bloqué vers 2,5 Ma l'exutoire aval du bassin et que celui-ci ait été remblayé jusqu'à hauteur du barrage volcanique, la Loire se déplaça vers l'est, dans une position intermédiaire entre celle du chenal plio-pléistocène et celle de la gorge actuelle (fig. 2). La relance consécutive des dynamiques fluviatiles semble être à l'origine de la décharge grossière qui apparaît au sein du dépôt et dont le banc à galets du méandre de Farges représente la marque la plus caractéristique. L'accumulation de nouvelles coulées à l'exutoire du bassin (plateau de Rachat, fig. 2) devait cependant bloquer à nouveau l'évolution, et conduire à la mise en place de la séquence supérieure du remblaiement du bassin du Puy, portant à plus de $150 \mathrm{~m}$ l'épaisseur de la sédimentation. Ce n'est qu'après l'épanchement des coulées sommitales du plateau du Devès, vers $1 \mathrm{Ma}$, que le creusement de la vallée actuelle, une nouvelle fois déportée plus à l'est, a pu débuter.

Ainsi, si le volcanisme n'a jamais pu modifier le cours d'une évolution dictée par le fonctionnement tectonique régional et l'évolution du contexte bioclimatique, il apparaît néanmoins comme un facteur de perturbation notable. Les modifications de tracé du cours de la Loire, de même que les retards de son encaissement, en sont la preuve. Ces perturbations apparaissent par ailleurs dans le bassin du Puy comme la cause déterminante d'une sédimentation expliquée auparavant par la subsidence (Bout, 1960) ou d'importants mouvements néotectoniques supposés à l'origine d'une accentuation des contrastes morphologiques entre le horst et le bassin (Le Griel, 1991).

\section{LES PRINCIPAUX CHANGEMENTS HYDROGRA- PHIQUES ET LE VOLCANISME DU DEVÈS DANS LE HAUT BASSIN DE L'ALLIER}

\section{1) PRÉSENTATION}

Au nord du bassin houiller de Langeac, l'Allier prend une direction ONO entre le hameau de Truchon et LavoûteChilhac. II bifurque ensuite vers le nord pour rejoindre le bassin oligocène de Brioude. II évite ainsi le bassin oligocène de Paulhaguet, qui constitue une zone déprimée entre la terminaison septentrionale du Livradois à l'est et le horst de la Chomette à l'ouest (fig. 4). L'alignement de cette dépression dans le prolongement septentrional du bassin de Langeac a été souligné par Bout (1960). Cette disposition et l'existence d'une épaisse sédimentation plio-quaternaire l'ont conduit à envisager un passage de la rivière dans cette dépression au Villafranchien inférieur. 


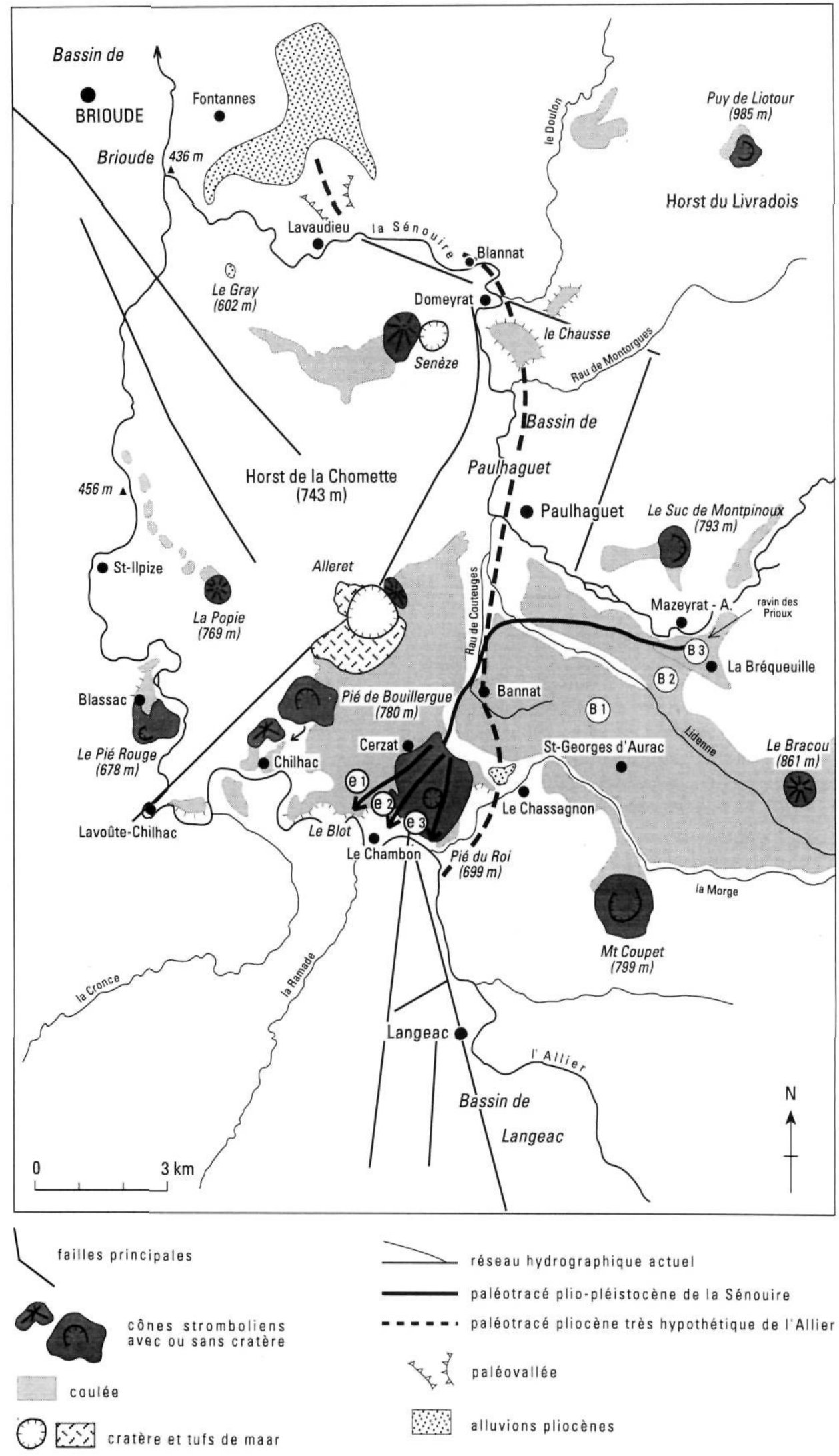

FIGURE 4. Évolution du système hydrographique dans le secteur Paulhaguet - Langeac - Brioude

Evolution of the hydrographic system in Paulhaguet - Langeac Brioude area. 
Une thèse a priori peu compatible a été ensuite émise par Grangeon et Pelletier (1964), puis élaborée par Marty (1965) et Grangeon (1967). C'est celle d'un ancien cours nord-sud de la Sénouire, venant se jeter dans l'Allier au sud de Cerzat, avant la volcanisation de la dépression et le déplacement de la rivière vers le nord. Cette interprétation a été confirmée par le levé aéromagnétique du Massif central (Roux et Sénaud, 1978) et précisée par de nouvelles données de terrain (Pastre, 1987). Un schéma cherchant à concilier les deux hypothèses et impliquant aussi un passage de l'Allier par la dépression a été proposé par Larue (1979 et-1982).

\section{2) L'HYPOTHĖSE D'UN ANCIEN TRACÉ DE L'ALLIER PAR LE BASSIN DE PAULHAGUET}

L'hypothèse d'un ancien tracé de l'Allier par le bassin de Paulhaguet est avant tout suggérée par la morphologie, mais l'analyse conjuguée de la géométrie des formes et des formations datées par les produits volcaniques la rend peu vraisemblable. L'existence d'un réseau hydrographique plio-pléistocène très encaissé à l'emplacement du cours actuel de l'Allier, ainsi que l'absence de preuves sédimentologiques plausibles au sein du bassin tend à faire prévaloir l'hypothèse de la pérennité du tracé actuel.

\section{a) Les preuves "trompeuses " de la morphologie}

L'alignement du bassin de Paulhaguet dans l'axe du bassin de Langeac a fait dire à Bout (1960), qu'elle constituait la "voie de passage logique de l'Allier". Sur le plan morphostructural, si l'alignement des deux bassins est net (fig. 4), il importe de remarquer que les deux structures sont néanmoins cloisonnées. Le môle gneissique de Mazeyrat $(671 \mathrm{~m})$ se poursuit en seuil au sud de Saint-Georgesd'Aurac-Gare $(600 \mathrm{~m})$ et réapparait en fenêtre entre les basaltes au nord de Cerzat.

D'un point de vue morphologique, il est vrai que la largeur moyenne du bassin de Paulhaguet $(5 \mathrm{~km})$ présente une ampleur plus que suffisante pour avoir pu offrir un passage à l'Allier. Si au nord, les gneiss de Domeyrat déterminent actuellement une topographie beaucoup plus encaissée, celleci a été fortement retouchée au Quaternaire. La morphologie antévolcanique reste compatible avec un passage de la rivière à un niveau d'encaissement moindre. Plus au NO, entre Blannat et Lavaudieu, la vallée actuelle de la Sénouire prend un profil évasé, qui peut être analysé comme l'héritage éventuel d'un passage antérieur de l'Allier. Enfin, le berceau situé au NE de Lavaudieu (fig. 4) peut être interprété comme une paléoforme prouvant le passage direct de la rivière vers Fontannes et le bassin de Brioude. Au sud, enfin, le couloir volcanisé et remblayé, emprunté actuellement par le ruisseau de Couteuges, constitue aussi une voie de passage potentielle dans la prolongation du bassin de Langeac.

\section{b) Les données sédimentologiques}

Aux deux extrémités du tracé envisageable, les alluvions du Chassagnon (Pastre, 1987) et celles de Fontannes (Bout, 1960 ; Larue, 1979 ; Pastre, 1987), représentent deux jalons pliocènes, mais cependant diachroniques (fig. 4). Les chailles rouges du Charmouthien des Causses démantelés du haut bassin de l'Allier et les galets de quartz filonien bien roulés qui les caractérisent permettent de les attribuer sans équivoque à l'Allier. Les alluvions à chailles du Chassagnon, appartiennent à la génération d'alluvions pliocènes anciennes, comparables aux alluvions de Pardines, près d'Issoire qui sont recouvertes par une coulée basaltique datée à 4,2 Ma \pm 0,3 Ma par K-Ar (Bout et al., 1966). Les alluvions de Fontannes, moins riches en chailles et qui contiennent déjà quelques basaltes, semblent plus tardives. Elles incorporent dans leur matrice résiduelle des quartz de ponces qui tendent à prouver leur postériorité par rapport aux émissions pyroclastiques rhyolitiques du Mont-Dore, datées vers $3 \mathrm{Ma}$ (Pastre, 1987). Ces dépôts paraissent engagés dans le tracé potentiel (fig. 4). Cependant, leurs positions respectives restent aussi compatibles avec un déplacement progressif de la rivière vers son lit actuel.

Le matériel siliceux du bassin de Paulhaguet, qui pourrait constituer un jalon intermédiaire indiscutable, n'est pas comparable. Les épandages résiduels développés sur l'Oligocène de la partie nord du bassin, de part et d'autre du ruisseau de Montorgues (fig. 4), comportent de nombreux quartz, mais il s'agit de galets de quartz d'origine métamorphique, généralement beaucoup moins roulés, émoussés et microfissurés. Ils sont attribuables à un alluvionnement local non datable, remanié et colluvionné. Si un matériel proche existe sous les coulées plio-quaternaires des environs de Domeyrat (Le Chausse, le Gourd), il ne contient pas non plus de chailles aptes à prouver un passage de l'Allier.

En ce qui concerne les formations plio-pléistocènes (sables, limons et argiles), contribuant au remplissage du bassin, rien ne permet de les attribuer à l'Allier, plutôt qu'à la Sénouire ou à des émissaires secondaires. L'existence de cortèges de minéraux lourds à grenat et staurotide, comparables à ceux de l'Allier, dans la zone d'apport de la Sénouire (Pastre, 1987), s'oppose à des cortèges très enrichis en staurotide ou en grenat. Ces derniers paraissent dans le premier cas révélateurs de facteurs d'altération (association quartz, muscovite, staurotide, limonite) et dans le second d'apports liés à des émissaires secondaires (association quartz, feldspaths, grenats).

c) La géométrie du réseau hydrographique plio-pléistocène

L'ensemble des données démontre l'encaissement prononcé du réseau hydrographique plio-pléistocène. À Truchon, les alluvions qui sont recouvertes par une coulée du Pié-duRoi datable d'environ $2 \mathrm{Ma}$ (Pastre, 1987 ; Baubron, in Fouris, 1989) se situent à $490 \mathrm{~m}$ (fig. 5 et 6 ), soit $90 \mathrm{~m}$ plus bas que les alluvions pliocènes du Chassagnon et seulement une dizaine de mètres plus haut que l'Allier actuel. Au sud du bassin de Paulhaguet, un des sondages de Bannat ( $\mathrm{n}^{\circ}$ d'inventaire BRGM 790-3x-0010) a traversé $41 \mathrm{~m}$ de sédiments fluvio-lacustres, puis une coulée de $23 \mathrm{~m}$ et $1 \mathrm{~m}$ de tufs, avant d'atteindre le socle vers $488 \mathrm{~m}$, soit à une altitude proche de l'altitude des alluvions de Truchon. En descendant la vallée de l'Allier, les alluvions sous-basaltiques de Chilhacvillage $(1,62 \pm 0,04 \mathrm{Ma}$, Cassignol in Couthures, $1982 ; 1,65$ $\pm 0,35$, Cantagrel in Bœuf, 1983) affleurent vers $490 \mathrm{~m}$, soit à environ une vingtaine de mètres au-dessus de l'Allier actuel, 
WNW

ESE

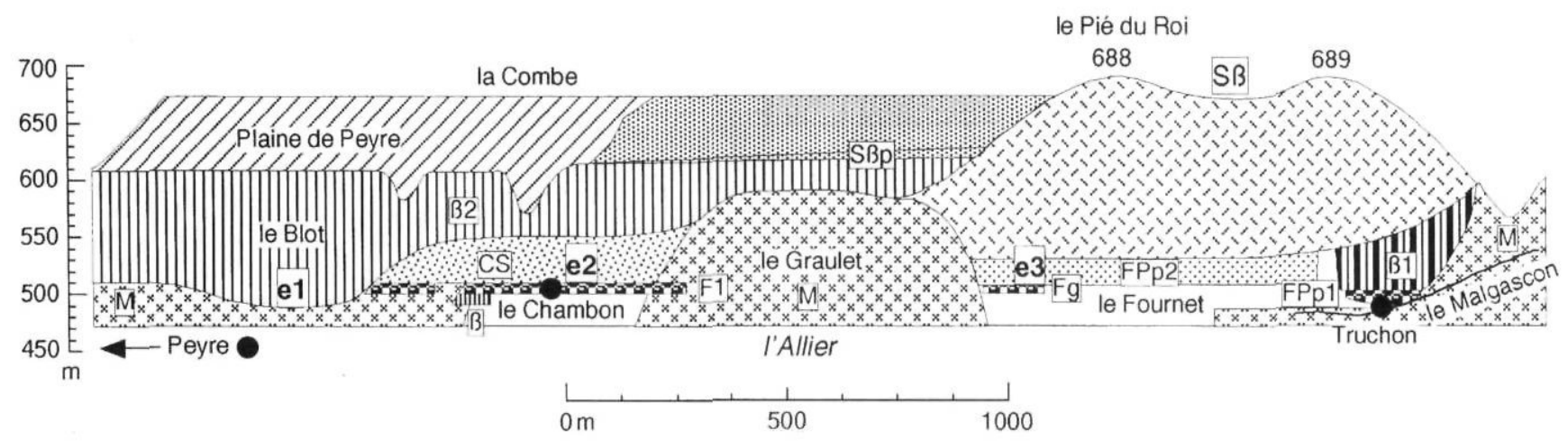

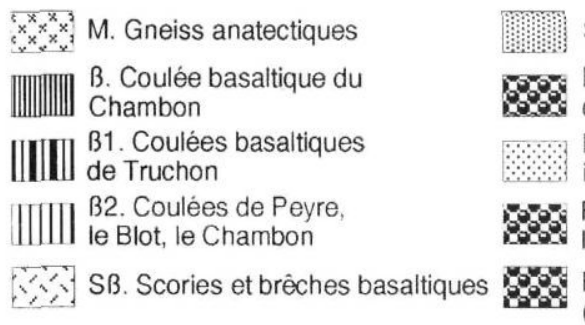

SBp. Scories de saupoudrage

FPp1. Alluvions sous-basaltiques de Truchon

FPp2. Sables à scories et basaltes infra scories du Piè du Rol

Fg. Alluvions à galets de basalte de la retombée sud du Pié du Roi F1. Terrasse du Chambon (post-basaltes)
FIGURE 5. Coupe schématique des formations plio-quaternaires en rive droite de l'Allier entre Truchon et Peyre.
Schematic section of the Plio-Quaternary formations on the left bank of Allier between Truchon and Peyre

S
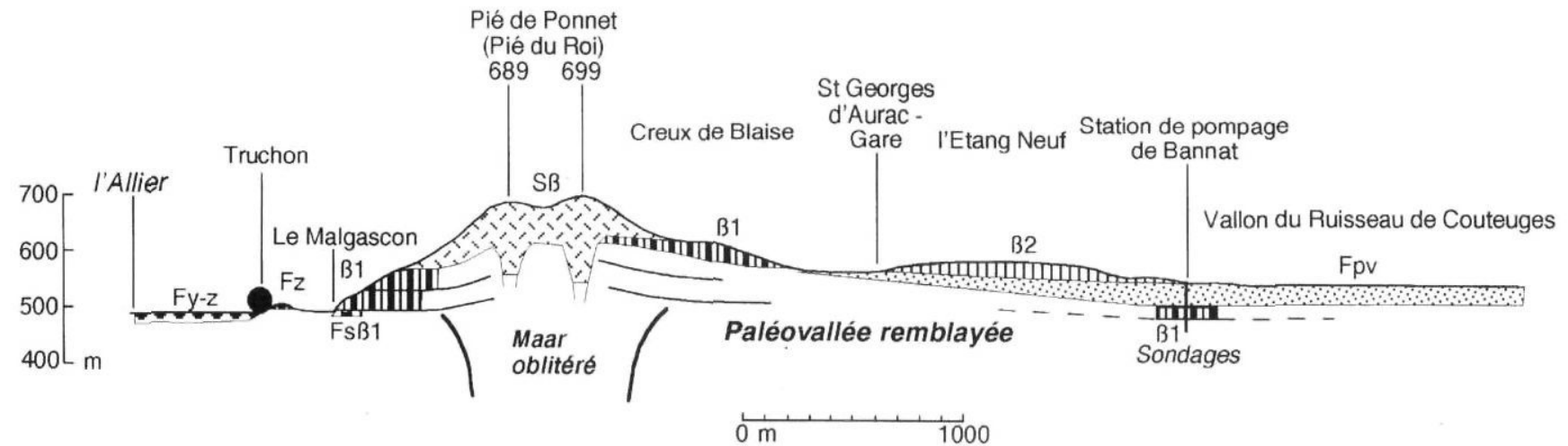

Kg FsB1 Alluvions sous-basaltiques 288 de Truchon et formations associées

IIII B1 Basaltes à nodules de Iherzolites

[N] SB Scories et brêches basaltiques

Fpv Formations fluvio-lacustres

du bassin de Paulhaguet
FIGURE 6. Coupe de la terminaison nord-occidentale du bassin de Langeac à la bordure méridionale du bassin de Paulhaguet.
Cross section between the $N-W$ part of Langeac graben and the southern part of Paulhaguet graben. 
celles situées sous la coulée inférieure de Blassac-Chambon $(2,15 \pm 0,11 \mathrm{Ma}$, Fouris, 1989) affleurent à $477 \mathrm{~m}$, soit à $26 \mathrm{~m}$ d'altitude relative. Ces données démontrent la coexistence d'un réseau hydrographique situé à un niveau d'encaissement similaire dans le sud du bassin de Paulhaguet et la vallée de l'Allier vers $2 \mathrm{Ma}$. Ce schéma est compatible avec un paléocours nord-sud de la Sénouire; par contre, il apparaît peu conciliable avec un passage de l'Allier par la dépression. Comment concevoir à la fois cette translation et l'existence d'une vallée bien incisée entre Truchon et Vieille-Brioude ?

Au nord du bassin de Paulhaguet, les alluvions sousbasaltiques des environs de Domeyrat se situent par ailleurs à plus de $550 \mathrm{~m}$, alors que le cours actuel de la Sénouire avoisine les $500 \mathrm{~m}$, altitude minimale de toutes les formations antéquaternaires du secteur. Si l'on admet le passage de l'Allier par la dépression, cette disposition à contrepente ne peut s'expliquer qu'en faisant intervenir des mouvements tectoniques de plusieurs dizaines de mètres de rejeu, ce dont le terrain ne fournit pas la preuve.

\section{d) Discussion}

Ces données convergent pour rendre improbable un passage de l'Allier par le bassin de Paulhaguet lors de la phase de volcanisation principale du secteur à la frontière plio-pléistocène.

La seule hypothèse qui puisse être envisagée éventuellement est celle d'un passage antérieur, lors du Pliocène inférieur ou moyen; mais on a vu le caractère aléatoire de la position des alluvions du Chassagnon ou de Fontannes et la différence des alluvions à galets de quartz du bassin. Quant à la paléoforme de Lavaudieu, elle peut aussi avoir été favorisée par la lithostructure des terrains houillers dans laquelle elle s'inscrit. Développer cette hypothèse conduit à expliquer l'abandon du tracé par le bassin de Paulhaguet et le creusement du tronçon Truchon - Vieille-Brioude. La volcanisation du bassin, en particulier la mise en place du Pié-du-Roi, étant plus tardive, il ne semble pas possible d'invoquer un barrage volcanique pour expliquer ce changement de cours. Quant à la tectonique, invoquée par Bout (1960) et Larue (1979 et 1982), elle semble insuffisante à expliquer entièrement le phénomène par rapport à la capacité compensatrice de creusement de l'Allier durant cette période de forte incision. Seul un phénomène de capture semble pouvoir être invoqué, mais il faut alors expliquer le creusement du tronçon Truchon Vieille-Brioude. L'intervention d'un affluent secondaire préfigurant le cours de l'Allier et collectant les eaux du rebord nordoriental de la Margeride constitue la seule solution envisageable, mais rien n'en apporte la preuve.

L'hypothèse d'un cours fixé dès le Néogène reste jusqu'à présent la solution la plus en accord avec les faits. Les preuves d'un passage ancien de l'Allier semblent d'ailleurs exister le long du tronçon Truchon - Vieille-Brioude. À Chilhac, les sables fluviatiles du Terret, qui se situent à une soixantaine de mètres d'altitude relative, soit près de quarante mètres plus haut que les alluvions sous-basaltiques du village, datent probablement de la césure Pliocène moyen - Pliocène supérieur (Pastre, 1987). À Coste-Cirgues, les galets de quartz du Gray (fig. 4), signalées par Grangeon (1961) ma- térialisent un niveau alluvial résiduel ancien (Mio-Pliocène ?) attribuable à l'Allier, qui domine le fond de vallée de près de $150 \mathrm{~m}$. Enfin, la morphologie en berceau de la section médiane de la vallée, située par l'ensemble des auteurs au Pliocène, paraît difficilement attribuable à une rivière de taille inférieure à l'Allier.

\section{3) Les changements de cours de la Sénouire}

Pour Grangeon (1967), “ les sédiments villafranchiens du bassin de Paulhaguet se sont déposés dans un lac formé à la suite du barrage de la Sénouire par des coulées de laves émises par le Pié-du-Roi et par un autre volcan de la région de Saint-Privat-du-Dragon ». Cette hypothèse peut être élaborée et discutée à la faveur de nouveaux éléments.

\section{a) L'existence d'une paléovallée remblayée dans l'axe du ruisseau de Couteuges}

L'existence d'une paléovallée remblayée dans l'axe du ruisseau de Couteuges est établie par plusieurs faits. Les sondages de Bannat constituent le premier indice d'une telle structure. Les $65 \mathrm{~m}$ de sédiments et de produits basaltiques traversés par le plus profond d'entre eux (voir plus haut), mettent la base de l'ancien thalweg à moins de $488 \mathrm{~m}$, alors qu'au NE de Cerzat, le socle, qui apparaît en fenêtre sous les basaltes des environs du Buisson, matérialise un paléoversant qui remonte à plus de $600 \mathrm{~m}$ (fig. 4).

À l'ouest du Chassagnon, on peut observer un ancien ravin fossilisé par des tufs phréatomagmatiques palagonitisés et une coulée de basalte attribuables au Pié-du-Roi (Marty, 1965 ; Grangeon, 1967). Ce paléo-ravin à profil tendu, qui correspond peut-être à un ancien cours de la Morge, prolongé sous la coulée du Coupet, se dirige suivant un axe SE-NO qui accrédite l'existence de la paléovallée vers l'ouest. Les données aéromagnétiques de Roux et Sénaud (1978) confirment ces résultats. L'affleurement du socle vers $575 \mathrm{~m}$ dans le secteur du Chassagnon et vers $625 \mathrm{~m}$ au nord de Cerzat, délimite la zone de passage de la rivière au nord du Pié-duRoi et situe son exutoire entre Jozan et Truchon (fig. 4).

\section{b) Les tracés possibles de la zone avale de la paléovallée}

L'importante volcanisation du secteur rend difficile la localisation de la confluence de la paléovallée. Grangeon (1967) situe cet exutoire au Blot, entre le Chambon et Peyre (fig. 4 et 5 , e1). Cette hypothèse est toutefois discutable. Si la coulée du Blot épouse dans ce secteur une nette concavité (fig. 5), il est probable que cette forme n'est liée qu'à un vallon secondaire. On observe seulement un tuf phréatomagmatique reposant sur le socle à l'ouest de l'abrupt et l'absence d'alluvions au contact ne constitue pas un indice favorable. Par ailleurs, l'altitude du contact, située vers $500 \mathrm{~m}$, ne pourrait s'expliquer qu'en faisant intervenir des mouvements tectoniques décamétriques qui, bien que possibles, ne sont pas prouvés. La possibilité d'une sortie au nord du Chambon (fig. 5 , e2), peut être envisagée. Les colluvions sableuses qui dominent le hameau pourraient masquer un remblaiement alluvial de plus de $20 \mathrm{~m}$ à la sortie de l'exutoire. L'éventualité d'une confluence à la base du système volcanique du Piédu-Roi (fig. 4 et 5), semble toutefois la plus probable. Elle 
pourrait s'être réalisée vers Truchon, au sud de l'emplacement actuel du Pié-du-Roi (fig. 5, e3), ou au nord à l'est de Cerzat, cette hypothèse impliquant alors un coude de l'Allier sous le volcan et une combinaison des tracés e 2 et e3, ce dernier, emprunté par l'Allier, se faisant alors du sud vers le nord. Au NE du Chambon ("Le Graulet "), le socle remonte à la faveur d'une faille et un peu plus à l'est, il vient en contact abrupt avec les scories du Pié-du-Roi. (fig. 5). Ce contact matérialise l'existence d'un paléoversant, fossilisé par les scories du Pié-du-Roi, alors que sur le versant est du volcan à Truchon, ses coulées basales ont aussi été contenues dans une zone déprimée (490 m), enserrée par le socle qui remonte latéralement à plus de $570 \mathrm{~m}$.

c) Le barrage de la paléovallée par l'appareil volcanique du Pié du Roi

Ces données tendent à démontrer que le Pié-du-Roi est inscrit dans une zone effondrée qui prolonge le graben de Langeac. L'existence d'alluvions à la base sud du volcan, présume de leur prolongement sous le cône. À l'ouest, les sables recouverts par les scories du “Bois-du-Roi »viennent buter contre les gneiss du “Graulet » (fig. 5). À l'est, la coulée basale de Truchon recouvre des alluvions composées de sables et de cailloutis, qui matérialisent un paléochenal d'axe NO qui semble pénétrer sous le cône. La présence d'enclaves d'argile et de graviers roulés dans le basalte montre que le conduit éruptif, situé plus au nord a intercepté des formations fluvio-lacustres pénécontemporaines de l'épisode phréatomagmatique marquant le début de l'activité du volcan.

La géométrie et la nature des formations du volcan contribuent par ailleurs à éclaircir le rôle déterminant de ce dernier dans l'évolution sédimentaire du bassin. L'activité phréatomagmatique initiale, à l'origine des tufs de déferlantes basales jalonnant les paléothalwegs (Truchon, le Fournet, le Chassagnon...), semble avoir pris directement naissance dans la zone de confluence, où elle a sans doute déterminé un diatrème débouchant vers $490 \mathrm{~m}$ (fig. 6), puis elle a été rapidement supplantée par des épisodes effusifs à l'origine des coulées de Truchon et de la coulée basale de Bannat. La paléovallée a dès lors été obstruée et la sédimentation fluvio-lacustre a pu commencer dans le bassin de Paulhaguet. La poursuite de l'activité effusive, à l'origine de la coulée du Chassagnon et des coulées nord du volcan, a ensuite fait place à l'édification du cône de scories $(698 \mathrm{~m})$, qui a définitivement barré la paléovallée (fig. 6). La seule voie de passage restant au sud, étant fixée par le toit de la petite coulée située à l'ONO du Chassagnon (585 m, fig. 4), la rivière a du se déverser vers le nord où la zone déprimée intra-oligocène lui ouvrait la voie.

d) Le remplissage fluvio-lacustre du bassin de Paulhaguet et le déversement de la Sénouire vers le nord

Les arguments avancés par Grangeon (1967) fixent les grandes lignes de l'évolution fluvio-lacustre de la dépression de Paulhaguet après sa fermeture et accréditent le franchissement par la Sénouire des reliefs des environs de Domarget et son déversement dans la vallée du Doulon pour aboutir à son cours actuel (fig. 4). Cependant, l'histoire volcano-sédimentaire de la dépression semble avoir déterminé une évolution plus complexe. L'existence d'une première phase de sédimentation importante après la mise en place du barrage est très probable, mais elle semble s'être poursuivie par une succession d'épisodes de creusement et de sédimentation, consécutifs à la mise en place des coulées orientales du secteur.

Les limons sableux sous-basaltiques à muscovite qui marquent le sommet de la sédimentation fluvio-lacustre affleurent vers $585 \mathrm{~m}$ au Viallard (2 km à l'ouest de Saint-Georges d'Aurac) ainsi qu'au nord de Bannat (fig. 4). Ceci confère au remblaiement une épaisseur maximale d'une centaine de mètres dans l'axe du ruisseau de Couteuges et est compatible avec le franchissement par la Sénouire des buttes gneissiques des environs de Domarget, dont les sommets ne dépassent pas actuellement $570 \mathrm{~m}$. Dans ce secteur, les alluvions sous-basaltiques du Chausse pourraient marquer le passage possible de la rivière vers $565 \mathrm{~m}$.

Après la percée, la dépression semble avoir connu une évolution polyphasée en liaison avec le volcanisme du secteur. L'altitude de base de la coulée de Saint-Eugénie-de-Villeneuve (fig. 4, B1) à Bannat, située à moins de $550 \mathrm{~m}$, montre que le déblaiement du remplissage initial était déjà amorcé lors de l'épanchement. Auparavant, la Sénouire, sortant de sa vallée encaissée, décrivait probablement une courbe, sensiblement calquée sur le cours aval actuel de la Lidenne (fig. 4). La coulée l'a probablement contrainte à recreuser son cours au nord. Son chenal a ensuite été oblitéré par la coulée du Bracou (B2, fig. 4), qui l'a obligé à recreuser son lit à la marge nord de cette coulée, à l'emplacement approximatif du tronçon actuel Esfacy-Paulhaguet. La dernière étape semble liée à l'épanchement des trois coulées du ravin des Prioux (B3, fig. 4), qui épousent la forme de l'ancienne vallée à son débouché dans le bassin au nord de la Brequeuille (Grangeon, 1967 ; Pastre, 1987). Ces coulées - du moins les supérieures - ont conduit la Sénouire à se déplacer sensiblement vers le nord, au sud de l'emplacement actuel de Mazeyrat-Aurouze et au niveau de sa vallée actuelle.

Les minéraux téphriques du Mont-Dore, remaniés dans les sédiments de la dépression, tendent à placer l'essentiel de l'évolution à la charnière plio-quaternaire. La caractérisation d'importantes injections à feldspaths alcalins, amphibole brune (kaersutite), oxydes de Fe-Ti et sphène dans le corps principal du remplissage, laisse supposer une corrélation avec le paroxysme d'activité plio-pléistocène du Mont-Dore vers $2 \mathrm{Ma}$ (Pastre, 1987). L'augmentation de la fréquence des clinopyroxènes verts du Mont-Dore (augite) dans les sédiments les plus tardifs (ravin des Prioux) peut s'accorder avec la poursuite des phénomènes volcano-sédimentaires durant le début le Pléistocène inférieur, voir au delà.

\section{L'ÉVOLUTION DU RÉSEAU HYDROGRAPHIQUE À LA BORDURE OCCIDENTALE DE LA LIMAGNE DU SUD ET LES VOLCANISMES DU CÉZALLIER ET DU MONT-DORE}

1) Le changement de cours de l'Allagnon et le volcanisme basaltique pliocène du Cézallier

L'existence d'un ancien cours de l'Allagnon, fossilisé par les laves pliocènes du Cézallier, à été établie par Thonat 
FIGURE 7. Reconstitution du paléo-tracé de l'Allagnon d'après la position des coulées et des alluvions sous-basaltiques.

Reconstitution of the paleo-course of the Allagnon according to the position of the lava-flows and of the subbasaltic alluvium.

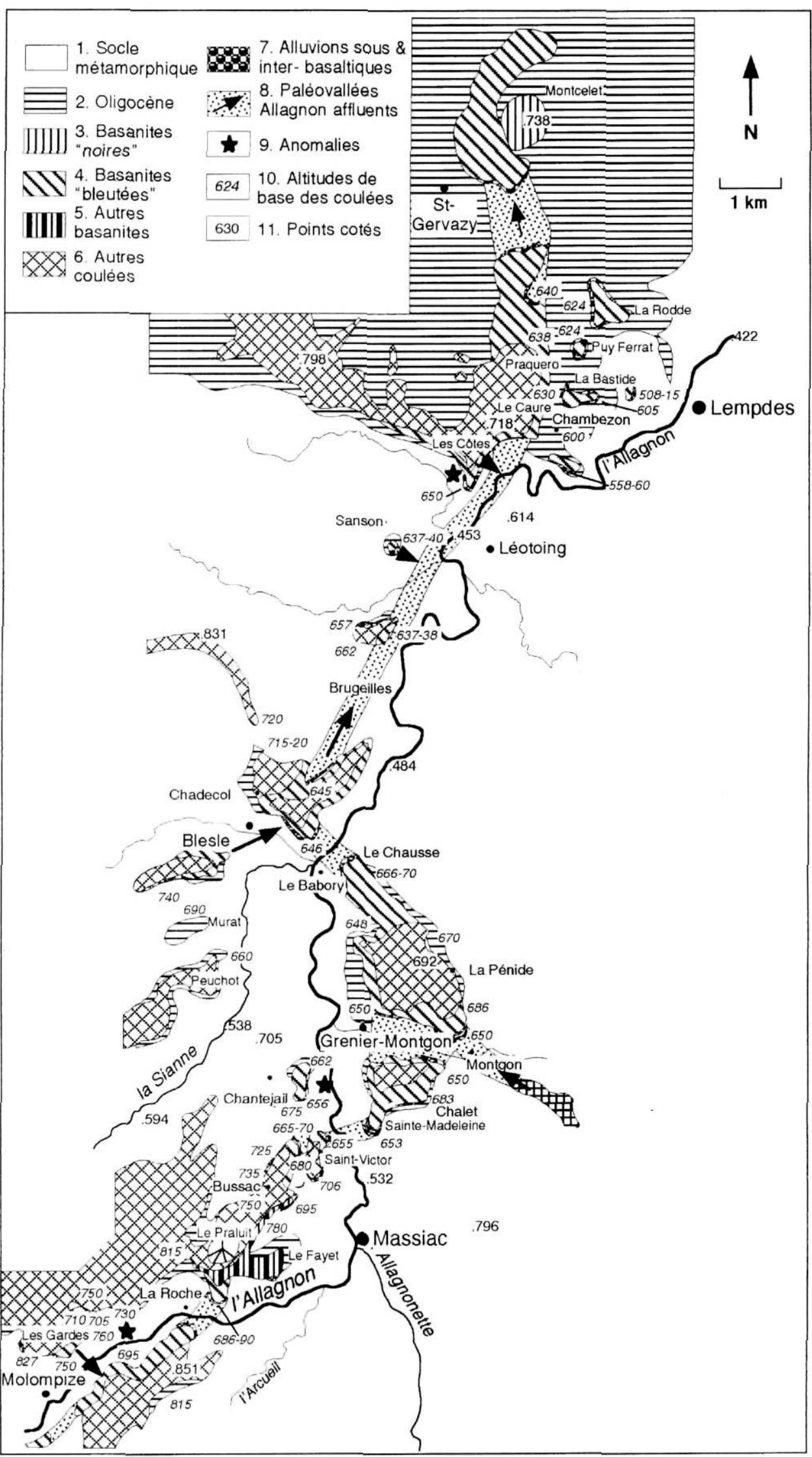


(1966). La réalisation d'un travail détaillé sur le cours moyen et inférieur de la rivière (Gablier, 1994) a permis d'en préciser les modalités, tout en en modifiant localement le tracé. Ce sont les "basanites noires " du Cézallier (A. Thonat, 1966 et 1969), datées à 5,35 $\pm 0,15$ Ma par K-Ar à Saint-Gervazy (Cantagrel et Thonat, 1976), qui ont les premières figé ce cours, emprunté par des coulées ultérieures de "basanites bleutées ". La carte des coulées et des alluvions pliocènes (fig. 7) montre que l'Allagnon suivait un cours étroit, orienté SSO-NNE jusqu'à Bussac, pour amorcer une courbe vers I'ENE au nord de Massiac. Entre la chapelle de SainteMadeleine et le Babory, la paléovallée, située en rive droite de la vallée actuelle, prenait une orientation subméridienne, tout en s'élargissant dans les sédiments oligocènes. Elle s'incurvait ensuite vers le NO en direction de Blesle, pour s'infléchir rapidement en direction du NNE et gagner la bordure occidentale de la Limagne, à l'ouest de Chambezon. Dans ce tronçon, pauvre en témoins, il est probable que la rivière passait à la marge de la Chaux de Brugeilles, où un paléoversant correspondant au flanc de la vallée est identifiable. Mais elle évitait sans doute le secteur de Sanson, dont la coulée, située trop haut, semble jalonner une paléovallée adjacente (Gablier, 1994). Le dernier tronçon, qui empruntait la bordure occidentale de la Limagne, suivait une direction subméridienne bien différente du cours aval actuel. Plusieurs lambeaux d'alluvions sous-basaltiques le jalonnent de la latitude de Chambezon à Saint-Gervazy (fig. 7) et il est probable que la confluence avec l'Allier se situait alors aux environs de Saint-Germain-Lembron.

Dans cette reconstitution paléogéographique, la position topo-altimétrique de plusieurs coulées ou le contenu sédimentologique de leurs alluvions sous-basaltiques permettent par ailleurs d'établir l'existence probable de cinq paléoaffluents (fig. 7). Le secteur entre Molompize et Massiac semble avoir connu un important exhaussement du lit de la rivière vers $4 \mathrm{Ma}$. L'empilement des coulées séparées par des alluvions dépasse en effet une centaine de mètres à la Roche. Ce phénomène semble avoir été par contre beaucoup plus limité vers l'aval, où les alluvions intercalées entre les coulées disparaissent rapidement après Grenier-Montgon.

La position du cours actuel montre que pendant tout son parcours dans le socle, la rivière a réentaillé alternativement sa vallée de part et d'autre des coulées jalonnant la paléovallée. À son débouché dans la Limagne, elle a par contre abandonné son cours $\mathrm{N}$-S, pour adopter rapidement une direction proche de l'actuelle. Si la position syn- ou postpaléovallée des buttes basaltiques de la Bastide, du Puy Ferrat et de la Rodde reste controversée (Le Griel, 1991; Gablier, 1994), celle des lambeaux " tardifs » du Cimetière et du "Concasseur » de Chambezon (Thonat, 1966 ; Pastre, 1987 ; Gablier, 1994), montre clairement que la rivière avait pris une orientation SO-NE lors du Pliocène moyen.

L'intérêt de ce paléotracé dépasse son caractère paléogéographique. II montre l'existence au Pliocène inférieur d'un tracé de l'Allagnon incisé dans le socle, proche de l'actuel, et atteste de l'existence d'un réseau hydrographique bien organisé, qu'il est possible de corréler aux traces plus discrètes évoquées précédemment pour la Loire ou le haut Allier.

\section{2) Les modifications de tracés dans la région des Couzes}

Le système hydrographique constitué par les rivières que sont les «Couzes " de Valbeleix, Pavin et Chambon a connu des modifications relativement importantes au Quaternaire. Les changements de tracé peuvent être retracés par l'étude conjointe des paléoformes et des formations liées au volcanisme du Mont-Dore qui leur sont associées. Ces données permettent de montrer qu'au Plio-Pléistocène, la Couze Chambon confluait avec l'Allier au SE de Pardines juste après avoir collecté les eaux de la Couze de Valbeleix, dont le cours aval préfigurait alors celui de l'actuelle Couze Pavin. La Couze Pavin, était alors tributaire de la Couze Chambon qu'elle rejoignait dans le secteur du Pont-de-Rivalet (fig. 8 a).

\section{a) L'ancien cours de la Couze Pavin à l'amont de Saurier}

L'ancien cours de la Couze Pavin à l'amont de Saurier peut être établi grâce à la répartition géographique des formations volcaniques qu'il a canalisées et la conservation ultérieure de sa vallée morte entre Saint-Diéry et le Pont-de-Rivalet. Le trajet emprunté par les épiclastites mondoriennes de génération Sancy ("lahar de type Fontenille ", Besson et al., 1977 ; Besson, 1978 ; “ lahars de seconde génération » : Ly, 1982 ; Pastre, 1982 et 1987), démontre qu'à la fin du Pléistocène inférieur, la rivière poursuivait son cours vers l'ENE à partir de Saint-Diéry pour rejoindre la Couze Chambon à l'emplacement actuel du Pont-de-Rivalet (fig. 8 a et b). Cette paléogéographie est confirmée par les témoins épiclastiques qui jalonnent le tracé aval de la Couze Chambon jusqu'à l'Allier (fig. 8 b). Ce tracé semble ne pas avoir évolué beaucoup jusqu'à la mise en place de la coulée pyroclastique de Neschers $\left(0,58 \pm 0,02 \mathrm{Ma} \mathrm{par}{ }^{39 \cdot 40} \mathrm{Ar}\right.$, Lo Bello et al., 1987). Cette coulée de cendres et ponces et les faciès alluviaux qui la remanient se suivent, en effet, du Cheix au SO de Saint-Diéry jusqu'à l'Allier en suivant la Couze Chambon (Besson et al., 1977 ; Ly, 1982 ; Pastre, 1982 et 1987 - fig. 8 b). Ils sont par contre absents dans la vallée aval de la Couze Pavin, ce qui conforte cette hypothèse. La coulée de trachybasalte de Champeix, de peu postérieure $(0,5 \pm 0,1 \mathrm{Ma}$ par $\mathrm{K}$-Ar ; Bout, Frechen et Lippolt, 1966) et dont la pétrographie est proche de celle des laves du Cheix, semble avoir aussi suivi le même tracé.

L'évolution ultérieure est matérialisée par la vallée morte empruntée par la D. 978 entre La Bataille et le Pont-deRivalet. Cette belle paléoforme, signalée par Gachon et Bout (1935), est totalement surdimensionnée par rapport à son drainage actuel assuré par le ruisseau de Rivalet. Elle tend à montrer que la Couze Pavin a recreusé sa vallée après les événements volcaniques précités. Le changement de cours semble avant tout imputable à une capture par déversement, mais le rôle de phénomènes volcaniques ou épiclastiques non caractérisés à l'affleurement (col de la Bataille, par exemple) reste possible. Cette capture de la rivière par la Couze de Valbeleix a toutefois nécessité un travail des ruisseaux de Verneuge et des Rives, qui ont érodé les unités de l'Oligocène du petit graben de Cotteuges, les deux tracés des rivières principales étant distants de plus de $2 \mathrm{~km}$ (fig. $8 \mathrm{~b}$ ). La différence altimétrique des niveaux de creusement entre la paléovallée ( $50 \mathrm{~m}$ environ) et la vallée actuelle permet de 


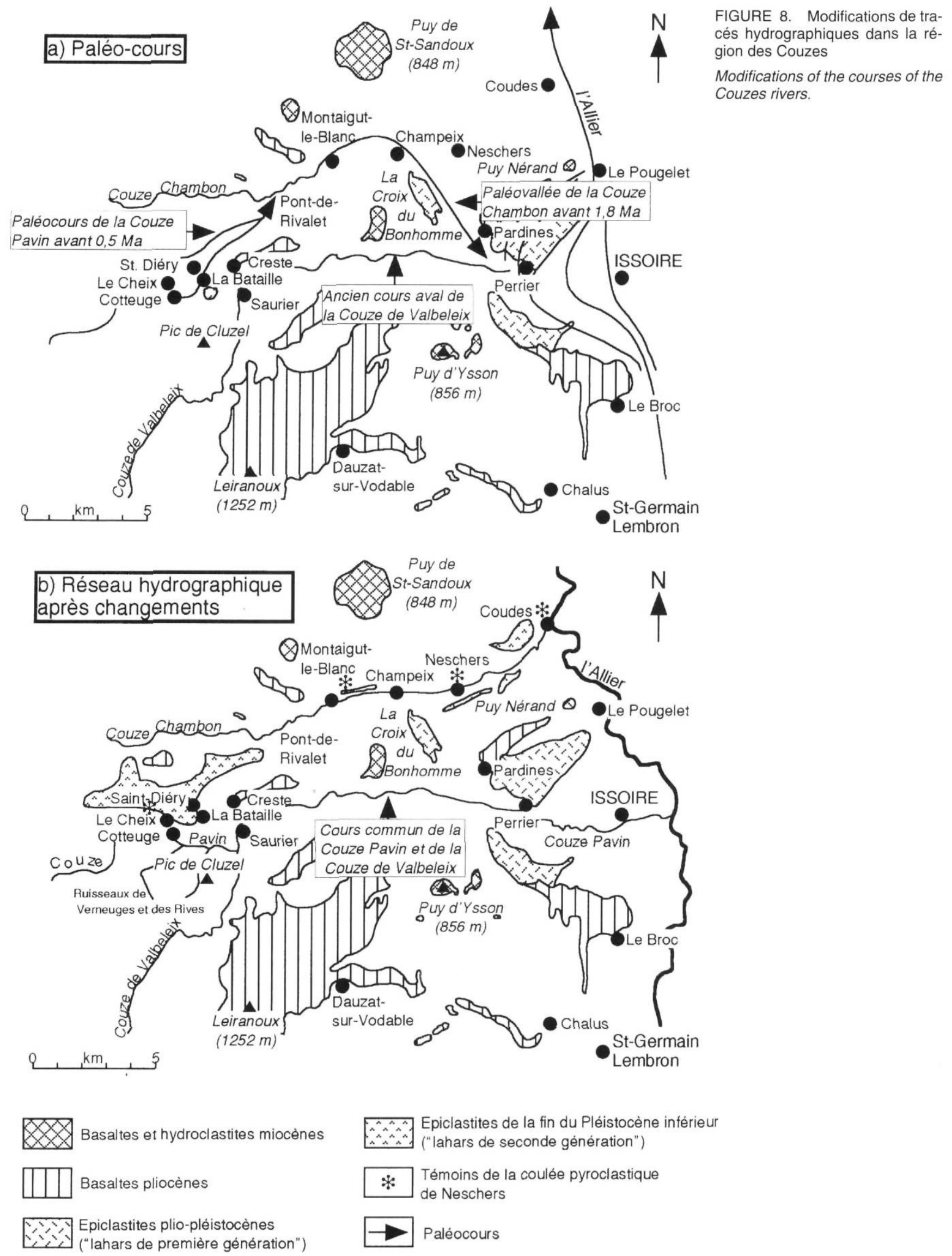


penser que ce changement est intervenu peu de temps après les phénomènes volcaniques du Pléistocène moyen.

b) L'ancien cours de la Couze Chambon à l'aval de Champeix

Les formations pyroclastiques et épiclastiques du Pliocène supérieur de la bordure orientale du Mont-Dore jalonnent un réseau hydrographique bien organisé qui préfigure le réseau actuel. Les grands lahars plio-pléistocènes issus des centres éruptifs situés au nord du col de la Croix-Morand ont en particulier suivi un trajet ONO-ESE proche des tracés actuels du Fredet et de la Couze Chambon, pour atteindre l'Allier dans le secteur d'Issoire (Besson, 1978 ; Ly, 1982 ; Morel, 1987 ; Pastre, 1987).

Au sud-est de Champeix, le plateau de la Croix-du-Bonhomme représente le témoin le plus aval de ces formations avant le plateau de Perrier (fig. 8 et 9). Les alluvions à ponces fibreuses rhyolitiques qui affleurent sur son versant sudoccidental vers $630 \mathrm{~m}$ (fig. 9) y marquent un premier stade d'encaissement datable d'environ $3 \mathrm{Ma}$ (âge ${ }^{39-40} \mathrm{Ar}$ des ponces : 3,07 $\pm 0,02 \mathrm{Ma}$, Féraud et al., 1990). Les lahars et l'avalanche de débris qui matérialisent son versant nord-est y définissent un second palier, inférieur à $570 \mathrm{~m}$. Son âge est proche de $2 \mathrm{Ma}$ (âge moyen des lahars : Cantagrel in Poidevin et al., 1984). L'organisation de ces dépôts montre qu'ils jaIonnent le flanc droit d'une paléovallée d'orientation NO-SE. La même orientation se retrouve dans le couloir étagé en contrebas entre Champeix et Pardines. Les affleurements situés à la partie basale de cette forme permettent d'observer des alluvions qui montrent qu'elle correspond bien à un tronçon de vallée abandonné. L'abondance de trachyphonolites et le cortège minéralogique (Pastre, 1987), prouvent que ces dépôts sont proches contemporains des émissions trachyphonolitiques groupées vers $2 \mathrm{Ma}$ (Cantagrel et Baubron, 1982). Un âge donné par la méthode des traces de fission de 2,03 $\pm 0,24 \mathrm{Ma}$ sur apatite, le confirme (Chambaudet et Couthures, 1980.
Ces éléments démontrent ainsi l'existence d'une paléovallée NO-SE entre Champeix et Pardines. Cette paléovallée correspond à l'ancien cours aval de la Couze Chambon, qui confluait alors avec l'Allier dans les environs de Perrier. L'exhaussement du lit de la rivière par les produits sous-saturés du Mont-Dore pourrait être en partie à l'origine du changement de cours. Le creusement important (50 $m$ environ) qui sépare ces dépôts des lahars de génération Sancy ( "lahars de seconde génération ", d'âge moyen $1 \mathrm{Ma}$ (Ly, 1982), et l'absence de recreusement dans l'axe de la paléovallée, suppose de toute manière une évolution contemporaine. La capture de la rivière par un affluent secondaire de l'Allier, drainant la bordure septentrionale de la mesa de Pardines, semble toutefois devoir être invoquée comme cause nécessaire, voire principale. Elle pourrait avoir été favorisée par des mouvements tectoniques, la basse vallée actuelle de la Couze étant inscrite sur des failles N 40-60 actives au Quaternaire.

Avant ce changement de cours, la Couze poursuivait sa route au SE de Pardines, pour confluer avec l'Allier dans le secteur de Perrier, après avoir reçu la Couze de Valbeleix. Ce tracé est attesté par le contenu sédimentologique des alluvions du Rif-du-Creux, qui affleurent à la partie occidentale du versant sud du plateau de Perrier (Aufrère, 1941; Ly, 1982 ; Pastre, 1987 et fig. 10). II s'agit en effet d'alluvions contenant essentiellement des produits volcaniques (basaltes, quartz et feldspaths, minéraux lourds, ponces fibreuses) et qui livrent quelques granites, staurotide et corindons, traceurs des apports du socle du versant oriental du Mont-Dore (Pastre, 1987). Leur richesse en ponces fibreuses les situe après $3 \mathrm{Ma}$. Leur affleurement sur le versant et leur épaisseur (+ de $25 \mathrm{~m}$ ), posent la question de leur engagement suivant une paléovallée s'engageant sous le plateau, à l'ouest du paléorelief formé par la coulée de Roca-Neyra. Cette hypothèse tentante, envisagée par Ly (1982), ne semble toutefois pas devoir être vérifiée. La coupe du versant nord du plateau (Pastre et al., 1983 ; Pastre, 1987), ne permet pas

NW

Plateau de la Croix du Bonhomme

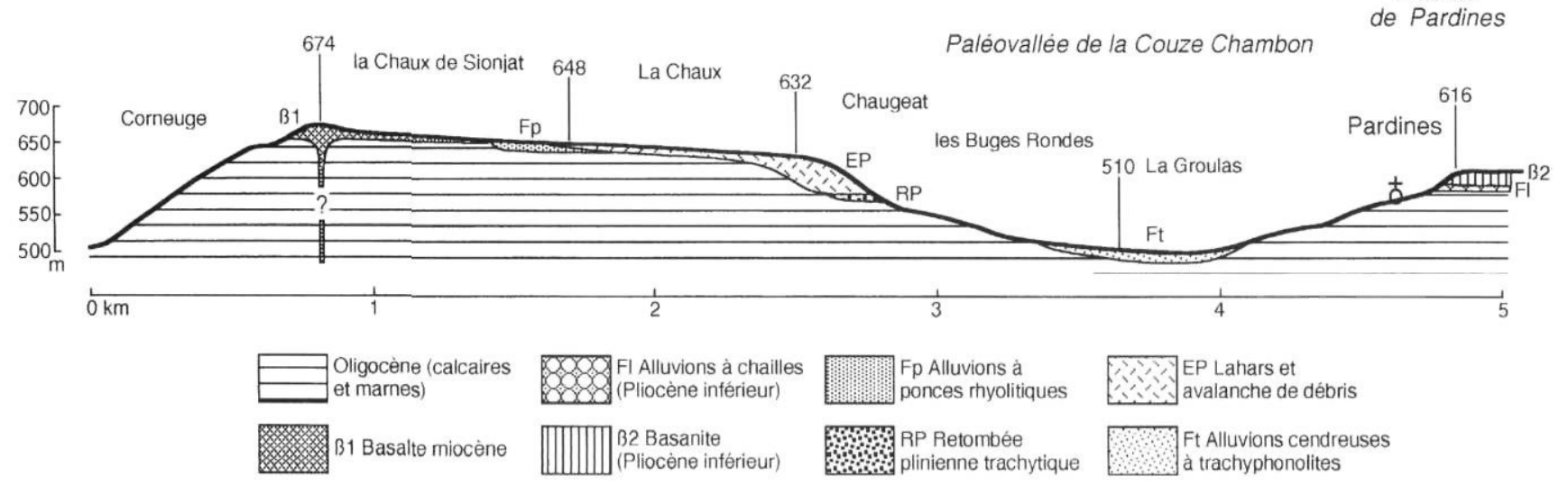

FIGURE 9. Coupe de la paléovallée de la Couze Chambon entre le plateau de la Croix du Bonhomme et le plateau de Pardines.

Cross-section of the paleovalley of the Couze Chambon river between la Croix du Bonhomme plateau and Pardines plateau. 
W

$$
\begin{gathered}
600 \\
E_{550} \\
E_{500} \\
E_{450} \\
E_{400 \mathrm{~m}}
\end{gathered}
$$

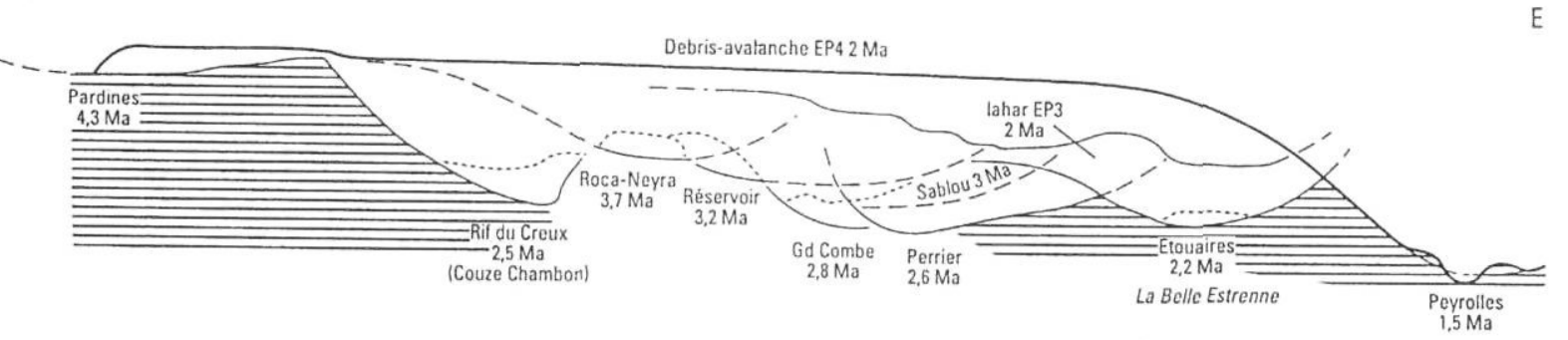

0

1

2

3

$4 \mathrm{~km}$

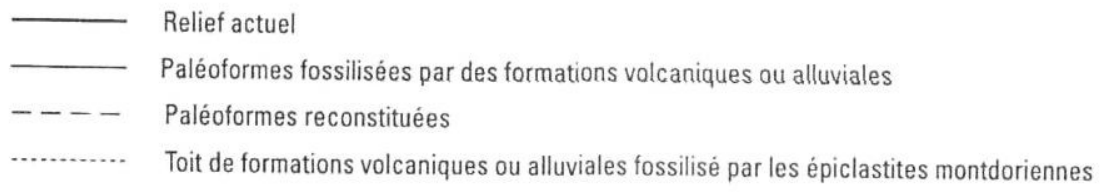

FIGURE 10. Lits successifs de l'Allier sur le versant sud du plateau de Perrier.

de définir un exutoire de ce tracé vers le nord, mais surtout, les formations situées plus à l'est, vers Perrier (celles à l'est de la coulée de Roca-Neyra, en particulier), témoignent comme on le verra d'apports de la Couze. L'hypothèse d'une accumulation dans une concavité et d'un tronçonnage $\mathrm{O}-\mathrm{E}$ de la coulée de Roca-Neyra par la rivière semble donc prévaloir.

\section{3) L'évolution du cours de l'Allier aux environs d'Issoire}

Les formations alluviales et volcaniques situées à l'ouest d'Issoire - la série du plateau de Perrier en particulier - permettent de reconstituer avec une bonne précision l'évolution complexe du cours de l'Allier depuis le Pliocène inférieur (Pastre, 1982b et 1987 ; Poidevin et al., 1984). La rivière, qui décrivait un important méandre, en a progressivement réduit la courbure pour atteindre son cours actuel (fig. 11). Ce dispositif a largement été contraint par la mise en place des coulées basaltiques pliocènes du Cézallier, qui ont détourné l'Allier de son tracé " naturel » originel dans les marnes oligocènes de la Limagne pour l'obliger à creuser des gorges dans le horst granitique de Saint-Yvoine.

Au début du Pliocène, l'Allier coulait aux environs de Pardines et continuait vers le nord par un tracé NO-SE, évitant le diatrème miocène du Puy Neyrand ou par un tracé plus méridien parallèle à la Limagne (fig. 11, stade "Pardines "). La coulée basaltique de Pardines, datée à 4,2 \pm 0,3 Ma par K-Ar (Bout et al., 1966), a fixé définitivement son cours en direction du horst granitique de Saint-Yvoine, en l'obligeant à recreuser son lit à la marge sud de la coulée (fig. 11). Cette dernière fossilise des alluvions à galets de quartz et chailles (Michel-Lévy et Munier-Chalmas, 1890 ; Pastre, 1987), dont la répartition témoigne d'un lit évasé, situé à $235 \mathrm{~m}$ d'altitude relative.

Au début du Pliocène moyen, les alluvions sousbasaltiques des Quinzes et de Roca-Neyra (altitude relative
Successive beds of the Allier on the southern side of the Perrier Plateau.

165-175 m), révèlent que le tracé passait dans le secteur du Broc, pour s'infléchir vers le nord-ouest en direction de Perrier (fig. 11, stade "Bergonne Roca-Neyra "). Vers 3,7 Ma (âge moyen des basaltes doléritiques de la bordure orientale du Cézallier: Cantagrel et Jamond in Ly, 1982), la coulée de Bergonne a obligé l'Allier à recreuser son lit à l'est du Broc, tout en continuant son tracé en direction de Perrier par un important méandre exagéré (fig. 11). L'encaissement s'est ensuite poursuivi à l'est de la coulée de Roca-Neyra (fig. 10, stade "Réservoir»). Après la mise en place de la grande coulée de ponces fibreuses rhyolitiques du Mont-Dore (fig. 10 et 11, stade Sablou de Lossa, vers $3 \mathrm{Ma}$ ), les alluvions de la Grand'Combe (fig. 10 et 11, stade " Grand'Combe » vers 2,8 Ma), montrent que le tracé a peu évolué durant la seconde moitié du Pliocène moyen, en dépit d'un creusement de plus de $60 \mathrm{~m}$. Ces alluvions sont en effet attribuables à l'Allier à l'aval de son ancienne confluence avec la paléo-Couze Chambon. Elles contiennent des galets de quartz filoniens et des grenats (Pastre, 1987) qui caractérisent des apports de l'Allier, alors que leurs minéraux volcaniques, qui proviennent pour l'essentiel du Mont-Dore, signalent les apports latéraux de la Couze.

Le stade suivant est matérialisé par les poudingues et les alluvions ponceuses classiques de Perrier. Ces formations, qui dénotent un contexte fluviatile présentant une charge réduite, suivi d'un remblaiement détritique important ( $35 \mathrm{~m}$ au minimum, plus de $50 \mathrm{~m}$ probablement), fossilisent la paléovallée contemporaine de l'Allier au nord de Perrier vers 2,6 Ma, et s'engagent sous le plateau en direction du nordest (fig. 10 et 11, stade "Perrier »). Leur pétrographie et leur minéralogie attestent, comme pour les précédentes, d'apports conjugués des deux rivières. Les apports de la Couze sont représentés par des sables cendreux à ponces fibreuses, riches en amphiboles brunes. Ceux de l'Allier par des sables quartzeux à silicates de métamorphisme et galets de quartz. 


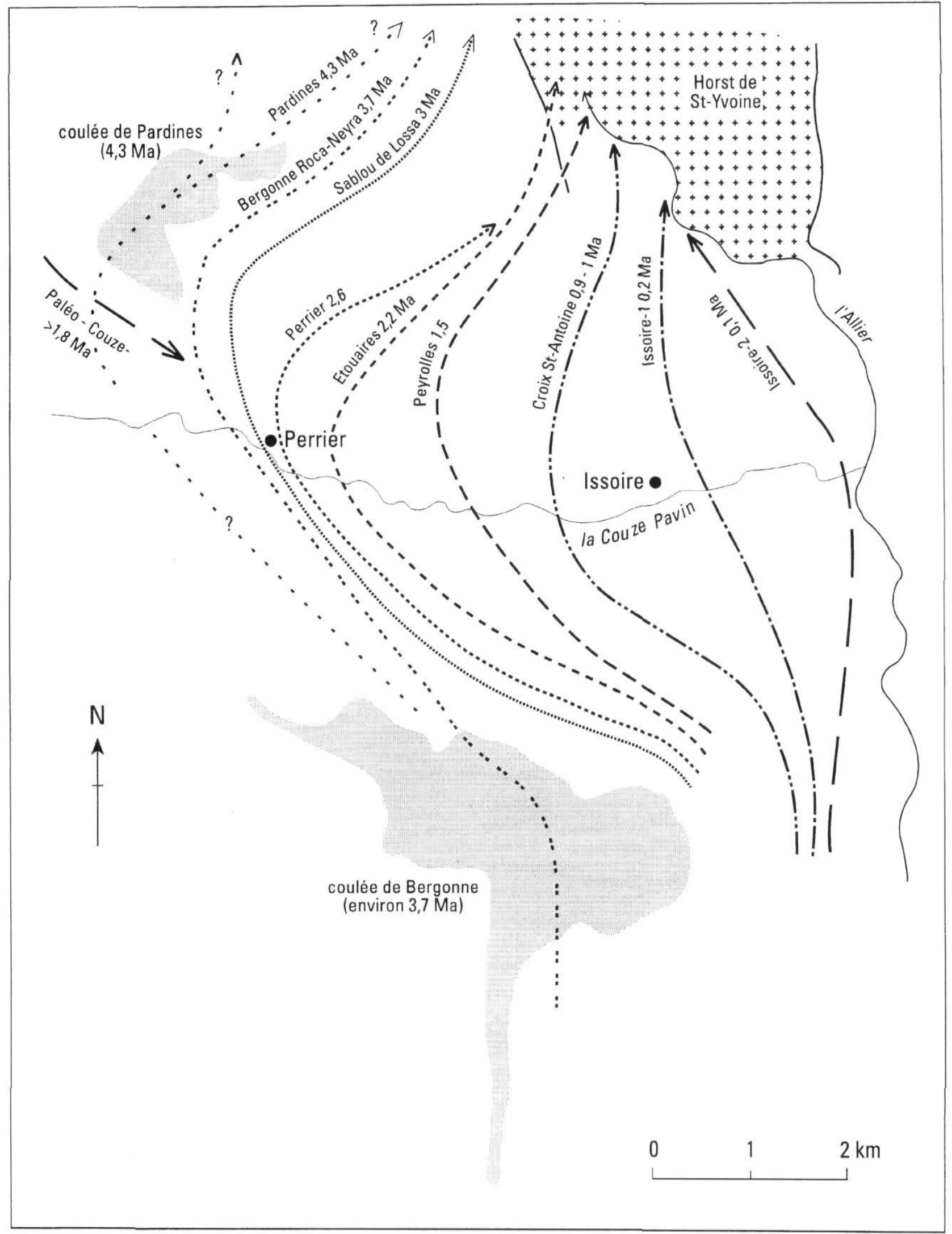

FIGURE 11. Tracés successifs de l'Allier dans le secteur de Perrier et Issoire. 
La migration de l'Allier vers l'est est ensuite bien caractérisée. Sur le versant sud du plateau de Perrier, le secteur de la Belle Estrenne permet de reconnaître ses alluvions et la forme de sa paléovallée, fossilisées par les épiclastites montdoriennes (fig. 10 et 11, stade "Étouaires "). À la base du versant est, la superposition directe du lahar inférieur sur ces mêmes alluvions permet de suivre son tracé SO-NE. Des pyroxènes de Iherzolites, liés au volcanisme contemporain du Devès, s'ajoutent aux traceurs antérieurs pour caractériser les apports longitudinaux de l'Allier, alors que des cortèges minéralogiques diversifiés signent les apports volcaniques synchrones du Mont-Dore, véhiculés par la Couze (Pastre, 1987).

Lors de la phase suivante, située vers $2 \mathrm{Ma}$ (Cantagrel in Poidevin et al., 1984), le comblement des thalwegs sur plus de $120 \mathrm{~m}$ d'épaisseur par les épiclastites du Mont-Dore a entraîné une modification importante des écoulements et la formation d'un véritable cône de déjection épiclastique dans la zone de confluence (Gachon et Bout, 1934). Pendant la mise en place des trois premiers lahars, la Couze et l'Allier ont recreusé rapidement leurs lits sans réatteindre leur niveau de creusement antérieur. Les bancs de galets repérables sur le versant sud du plateau de Perrier permettent une reconstitution de cette évolution (Pastre, 1987). Elle ne traduit toutefois pas encore de modifications paléogéographiques importantes et indique en particulier le recreusement d'un chenal après le dépôt du troisième lahar dans l'axe de la paléovallée de la Belle Estrenne (fig. 10, lahar EP3).

L'avalanche de débris supérieure EP4, qui inclut des blocs de téphrite de plusieurs centaines de mètres cubes, a par contre entraîné l'abandon définitif de la zone du plateau et un nouveau glissement de l'Allier vers l'est. II semble que lors du recreusement, la rivière ait exploité alors préférentiellement la zone de contact entre les épiclastites et l'Oligocène constituant le flanc droit de la paléovallée à la latitude d'lssoire. Au nord du plateau de Perrier, l'abandon de son tracé probable dans l'Oligocène au niveau du Pougelet pour son encaissement au sein du granite du horst, semble illustrer un phénomène de surimposition consécutif à la mise en place des épiclastites.

L'évolution paléogéographique ultérieure peut être reconstituée assez précisément grâce à la répartition des terrasses alluviales quaternaires et des produits volcaniques qui leur sont associés. Les alluvions cendro-ponceuses de Malbattu et du Creux-de-Peyrolles jalonnent la partie gauche du fond de la vallée de l'Allier au milieu du Pléistocène inférieur (stade Peyrolles, fig. 10 et 11). Les ponces trachytiques qu'elles contiennent ont donné des âges de 1,54 $\pm 0,13 \mathrm{Ma}$ par traces de fission sur sphène (Couthures et Pastre, 1983), 1,20 \pm 0,05 Ma par K-Ar (Poidevin et Cantagrel, 1984), 1,5 $\pm 0,01$ par ${ }^{39-40} \mathrm{Ar}$ (Lo Bello, 1988). Un kilomètre à l'est, les alluvions de la Croix-Saint-Antoine marquent le rebord externe d'une terrasse influencée par un épisode pyroclastique trachytique du Mont-Dore dont l'âge trace de fission de 0,91 $\pm 0,17 \mathrm{Ma}$ (Chambaudet et Couthures, 1981) est en bon accord avec les âges du lahar trachyandésitique et des alluvions à amphiboles auxquelles elles sont associées (Pastre, 1987). Enfin, si les stades suivants sont oblitérés, ceux de la fin du
Pléistocène moyen et du Pléistocène supérieur sont approximativement reconstituables par la géométrie des nappes alluviales (fig. 11). Ils montrent la poursuite du " glissement " de l'Allier vers l'est jusqu'à son cours subméridien actuel. Durant le Saalien (nappe Fwd) et le Weichsélien (nappes FX et Fy), cette évolution semble avoir été largement favorisée par les apports alluviaux latéraux « progradants " de la Couze Pavin.

L'Allier a donc connu dans ce secteur une migration de plus de $5 \mathrm{~km}$ depuis le Pliocène inférieur. Cette évolution constitue un exemple exceptionnel, mais il importe avant tout de retenir son caractère particulier, lié à la conjugaison des facteurs morphostructuraux et volcanologiques propres à ce secteur.

\section{CONCLUSION}

Les exemples présentés montrent le rôle déterminant du volcanisme plio-quaternaire du Massif central dans la modification de plusieurs tracés des bassins de la Loire et de l'Allier. Ils soulignent par ailleurs l'intérêt des datations radiométriques et des marqueurs téphrochronologiques dans les reconstitutions. Toutefois, l'analyse de terrain reste irremplaçable et sous-tend largement les interprétations proposées. Parmi les grandes questions paléogéographiques régionales, il semble que l'hypothèse d'une paléo-Loire plio-quaternaire affluente de l'Allier doive être abandonnée au profit d'un cours sub-méridien parallèle à l'actuel, fossilisé par les basaltes du Devès entre Arlempdes et le bassin du Puy.

Dans le haut bassin de l'Allier, l'éventualité d'un passage de l'Allier par le bassin de Paulhaguet ne trouve pas d'arguments décisifs. Elle ne semble guère constituer qu'une solution très hypothétique pour un cours pliocène, dont les traces manquent dans la dépression. Par contre, de nouvelles preuves d'un cours nord-sud de la Sénouire, barré ensuite par l'appareil volcanique du Pié-du-Roi sont proposées. Elles sous-tendent une évolution volcano-sédimentaire plio-pléistocène complexe, à l'origine d'un remblaiement fluvio-lacustre polyphasé et du déversement de la rivière vers le nord aux environs de la limite plio-quaternaire.

À la bordure occidentale de la Limagne du Sud, l'étude des alluvions sous-basaltiques de la vallée de l'Allagnon précise le paléotracé de la rivière au Pliocène inférieur, tout en signalant ses points communs et ses différences par rapport au tracé quaternaire. Plus au nord, l'étude des formations volcaniques et alluviales de la région des Couzes, permet de mettre en évidence différents paléotracés modifiés durant le Quaternaire. Si leur liaison avec le volcanisme pyroclastique et épiclastique du Mont-Dore étaye leur paléogéographie, elle semble par contre jouer un rôle subsidiaire dans leur évolution finale et l'hypothèse de captures semble prévaloir. Enfin, l'évolution spécifique du cours de l'Allier aux environs d'Issoire est analysée avec précision depuis le Pliocène grâce aux jalons fournis par le volcanisme.

Ces résultats soulignent la fixation générale des tracés depuis l'aube du Pliocène et confirment leur importante évolution durant le Pliocène moyen et supérieur. Le volcanisme plio-quaternaire apparaît comme un facteur de perturbation important, jouant un rôle déterminant dans l'aggradation 
sédimentaire des bassins volcanisés et réduisant le rôle directement attribuable à la tectonique. Toutefois, il ne provoque pas de bouleversement majeur des drainages dans un réseau hydrographique déjà très encaissé.

\section{REMERCIEMENTS}

Nous remercions les lecteurs de la revue, Serge Occhietti et Jean-Claude Dionne, pour leurs commentaires et suggestions fort utiles

\section{RÉFÉRENCES}

Ablin, D., 1985. Analyse pollinique de dépôts lacustres de Ceyssac (PlioPléistocène du Velay - Massif central français) : flore, végétation et climat. Thèse de $3^{\circ} \mathrm{cycle}$. Muséum national d'Histoire naturelle et Université Pierre et Marie Curie, Paris, $121 \mathrm{p}$.

_ 1991. Analyse pollinique des dépôts lacustres de Ceyssac, Plio-Pléistocène du Velay (Massif central, France). Cahiers de Micropaléontologie, N.S., 6-1: 21-38.

Aufrère, L., 1941. La colline de Perrier et les hypothèses eustatiques. Bulletin de l'Association des Géographes français, 138-139: 77-88.

Baulig, H., 1928. Le Plateau Central de la France et sa bordure méditerranéenne. Étude morphologique. A. Colin, Paris, 590 p.

Bellon, H., Brousse, R., Mervoyer, B. et Blais, S., 1972. Âge absolu du massif du Mont-Dore. Comptes rendus de l'Académie des Sciences, Paris, série D, $274: 2740-2743$.

Besson, J.C., 1978. Les formations volcaniques du versant oriental du massif du Mont-Dore (Massif Central français), feuille 1/25 000 Veyre-Monton 56. Thèse de $3^{\mathrm{e}}$ cycle, Université Blaise Pascal, Clermont-Ferrand., $167 \mathrm{p}$.

Besson, J.C., Ly, M.H., Cantagrel, J.M., de Goër de Herve, A. et Vincent, P.M., 1977. Une coulée de ponces post-villafranchienne sur le versant oriental du Mont-Dore. Comptes rendus de l'Académie des Sciences, Paris, série D. $284: 1875-1878$

Bœuf, O., 1983. Le site villafranchien de Chilhac (Haute Loire, France). Étude paléontologique et biochronologique. Thèse de doctorat d'État, Université de Paris VII, $253 \mathrm{p}$.

Boule, M., 1893. Description géologique du Velay. Thèse. Bulletin du Service de la carte géologique de France, $\mathrm{n}^{\circ} 28$.

Bout, P., 1960. Le Villafranchien du Velay et du bassin hydrographique moyen et supérieur de l'Allier. Thèse de doctorat d'État, Université de Paris, Impr. Jeanne d'Arc, Le Puy, $344 \mathrm{p}$.

- 1967. Observations sur le Villafranchien d'Auvergne et du Velay. Bulletin de l'Association française pour l'Étude du Quaternaire, 1: 3-64.

1980. La Loire supérieure affluent de l'Allier au Villafranchien? Almanach de Brioude : 131-147

Bout, P., Frechen, J. et Lippolt, H.J., 1966. Datations stratigraphiques et radiochronologiques de quelques coulées basaltiques de Limagne. Revue d'Auvergne, 80-4: 207-231.

Cantagrel, J.-M. et Thonat, A., 1976. Âge du volcanisme dans le Cézallier (Massif central français) et migration de l'activité volcanique en Auvergne. Comptes rendus de l'Académie des Sciences, Paris, série D, 282 : 1837. 1840

Cantagrel, J.M. et Baubron, J.-C., 1982. Chronologie des éruptions dans le massif volcanique des Monts-Dore (méthode potassium-argon). Implications volcanologiques. Géologie de la France (2), I, (1-2) : 123-142.

Chambaudet, A. et Couthures, J., 1981. Datations par traces de fission de gisements plio-pléistocènes de la périphérie des Monts-Dore (Massif Central, France). Comptes rendus de l'Académie des Sciences, Paris, série II, 293: $67-71$

Couthures, J., 1982. Contribution à la chronostratigraphie de formations pliopléistocènes du Massif Central (France). Thèse de $3^{E}$ cycle, Université Pierre et Marie Curie, Paris, $180 \mathrm{p}$.
Couthures, J. et Pastre, J.-F., 1983. Chronostratigraphie du Plio-Pléistocène d'Auvergne et du Velay : nouveaux apports des datations radiométriques et du paléomagnétisme. Bulletin de l'Association française pour l'Étude du Quaternaire, $2^{e}$ série, 13 : 9-18.

Crosnier-Leconte, J., 1948. Sur l'existence d'un ancien lit de la Loire dans la région d'Arlempdes (Haute-Loire). Comptes rendus sommaires de la Société géologique de France, $16: 347-349$.

1956. Remblaiement de l'ancien bassin supérieur de la Loire par les basaltes du Velay. Comptes rendus de l'Académie des Sciences, Paris, $242: 2746-2749$.

Defive, E., 1996. L'encaissement du réseau hydrographique dans le bassin supérieur de la Loire. Thèse de l'Université de Paris I Panthéon-Sorbonne, 549 p. et annexes.

Étienne, R., 1982. L'origine de l'alimentation du bassin d'Amphilhac (nord-ouest du Puy) et ses indications sur les changements hydrographiques et tectoniques récents au nord du horst du Devès. Bulletin du Laboratoire rhodanien de Géomorphologie, 11-12: 13-33

Féraud, G., Lo Bello, P., Hall, C.M, Cantagrel, J.M., York, D. et Bernat, M., 1990. Direct dating of Plio-Quaternary pumices by ${ }^{40} \mathrm{Ar} /{ }^{\beta 9} \mathrm{Ar}$ step-heating and single-grain laser fusion methods: The example of the Monts-Dore massif (Massif Central, France). Journal of Volcanology and Geothermal Research, $40: 39-53$.

Fouris, M., 1989. Les sites villafranchiens du Devès et les basaltes de la vallée de l'Allier: application de la méthode K-Ar. Thèse de l'Université Blaise Pascal, Clermont-Ferrandd., $206 \mathrm{p}$.

Gablier, F., 1994. Les étapes de creusement de l'Allagnon moyen et inférieur. Étude paléogéographique et géomorphologique. Mémoire de maîtrise de Géographie, Université de Paris I, $134 \mathrm{p}$

Gachon, L. et Bout, P., 1934. Le cône de déjection de Perrier. Bulletin de l'Association des Géographes francais, $80: 82-88$

1935. Perrier et la région des Couzes. Étude de géographie physique. Annales de Géographie, 250 : 349-370.

Grangeon, P., 1961. Aperçu des principales ressources du Brivadois. L'Almanach de Brioude : 99-103.

- 1967. Le cours villafranchien de la Sénouire dans le bassin de Paulhaguet et quelques remarques sur certains phénomènes volcaniques de la région de Cerzat (Haute-Loire, Massif central français). Bulletin de l'Association française pour l'Étude du Quaternaire, 2: 145-163.

Grangeon, P. et Pelletier, H., 1964. Ancien cours de la Sénouire, affluent de rive droite de l'Allier dans le bassin de Paulhaguet, Haute-Loire. Comptes rendus de l'Académie des Sciences, Paris, 258 : 1867-1869.

Larue, J.-P., 1979. Les nappes alluviales de la Loire et de ses affluents dans le Massif central et dans le sud du Bassin parisien: étude géomorphologique. Thèse de doctorat d'État, Université Blaise Pascal, Clermont-Ferrand, 2 vol., $544 \mathrm{p}$.

- 1982. Les enseignements climatiques et tectoniques fournis par l'étude des nappes alluviales de la Loire et de ses affluents. Revue de Géomorphologie dynamique, $4: 137-149$.

Le Griel, A., 1991. L'évolution géomorphologique du Massif central français. Essai sur la genèse d'un relief. Thèse d'État, Lettres, Université de Lyon II, 3 t., 660 p.

Lo Bello, P., Féraud, G., Hall, C.M., York, D., Lavina, P. et Bernat, M., 1987. ${ }^{40} \mathrm{Ar} /{ }^{39} \mathrm{Ar}$ step-heating and laser fusion dating of a Quaternary pumice from Neschers, Massif Central, France : The defeat of xenocrystic contamination. Chemical Geology (Isotope Geosciences Section), 66 : 61-71.

Lo Bello, P., 1988. Géochronologie par la méthode ${ }^{39} \mathrm{Ar}-{ }^{40} \mathrm{Ar}$ de ponces quaternaires contaminées - Exemple des ponces du Mont-Dore (Massif central français) - Utilisation d'un laser continu pour la datation de minéraux individuels. Thèse de doctorat de l'Université de Nice, $122 \mathrm{p}$.

Ly, M.H., 1982. Le plateau de Perrier et la Limagne du Sud: études volcanologiques et chronologiques des produits montdoriens (Massif central français). Thèse de $3^{\circ}$ cycle, Université Blaise Pascal, ClermontFerrand, $180 p$

Marty, J.P., 1965. Étude du volcanisme récent de la région de Saint-Georges $d^{\prime}$ Aurac (Haute-Loire). Thèse de $3^{e}$ cycle, Clermont-Ferrand, $100 \mathrm{p}$. 
Michel-Lévy, A. et Munier-Chalmas, 1890. - Compte-rendu de l'excursion du 19 septembre à Pardines, Perrier et Issoire. in Réunion extraordinaire de la Société géologique de France à Clermont-Ferrand et au Mont-Dore. Bulletin de la Société géologique de France, 18: 929-934.

Morel, J.M., 1987. Volcanologie du massif de l'Aiguiller (Mont-Dore, Massi central français) : étude pétrographique, dynamique, structurale et rhéologie des coulées de boue associées. Thèse de doctorat de l'Université Blaise Pascal, Clermont-Ferrand, $199 \mathrm{p}$.

Pastre, J.-F.,1982a. Contribution à l'étude des formations volcaniques et alluviales de la basse Couze Chambon (Massif central français). Bulletin de l'Association française pour l'Étude du Quaternaire., $2^{\circ}$ série, 9: 13-27.

1982b. Contribution à l'étude chronostratigraphique des formations pliocènes et pléistocènes des environs de Perrier et Issoire (Moyenne vallée de l'Allier, Massif central, France). Mémoire de Diplôme d'Études approfondies en Géologie du Quaternaire, Muséum national d'Histoire naturelle et Université Pierre et Marie Curie, Paris, $149 \mathrm{p}$.

1987. Les formations plio-quaternaires du bassin de l'Allier et le volcanisme régional (Massif central, France). Thèse de l'Université Paris VI. Mémoire des Sciences de la Terre, Université Pierre et Marie Curie, Paris, $n^{\circ} 87-32,733 p$.
Pastre, J.-F., Chambaudet A. et Couthures J., 1983. Nouveaux éléments d'interprétation stratigraphique et géodynamique des formations plio-pléistocènes de Perrier (Massif central, France). Comptes rendus de l'Académie des Sciences Paris, sér. II, $296: 79-84$.

Poidevin, J.L., Cantagrel, J.M. et Guerppa, 1984. Un site unique du Plio-Pléistocène en Europe : le Plateau de Perrier (Puy-de-Dôme). Revue des Sciences naturelles d'Auvergne, $50: 87-95$.

Robert, F., 1836. Mémoire géologique sur le bassin du Puy. Annales de la Société d'Agriculture du Puy, $27 \mathrm{p}$.

Roux, J. et Sénaud, G. 1978. Le levé aéromagnétique Massif central 1975. Étude de quelques anomalies caractéristiques liées au volcanisme et au socle. Thèse de $3^{\circ}$ cycle, Université de Clermont-Ferrand, $197 p$

Thonat, A., 1966. Le volcanisme de l'Est du Cézallier et la vallée fossile de l'Allagnon. Diplôme d'études supérieures, Université de Clermont-Ferrand, $177 \mathrm{p}$

1969. Les basanites noires de l'Est du Cézallier et la fossilisation de l'ancien réseau hydrographique de l'Alagnon. Revue des Sciences naturelles d'Auvergne, $35:$ 49-118. 